\title{
Handbuch für Exzellente Ökonometrische Effektmessung Öffentlicher Investition in Forschung und Entwicklung (Central Innovation Manual)
}

\author{
"CIM 2.0"
}

von

Dr. Thomas Alslev Christensen, Leiter der Abteilungen für Innovationspolitik und für Forschungs- und Innovationsanalyse, Hanne Frosch, Sonderberaterin, David Boysen-Jensen, Bereichsleiter,

Dänisches Ministerium für Forschung und weiterführende Bildung Agentur für Wissenschaft, Technologie und Innovation

Bredgade 40

1260 Kopenhagen $\mathrm{K}$

Dänemark

www.ufm.dk

22-05-2014 


\section{Inhaltsverzeichnis}

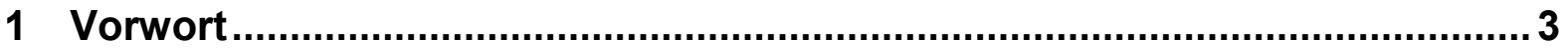

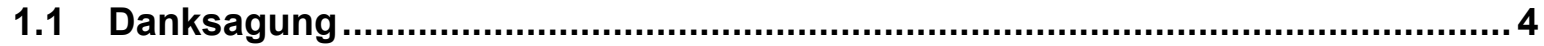

2 Teil I - Was ist das CIM (2.0)?

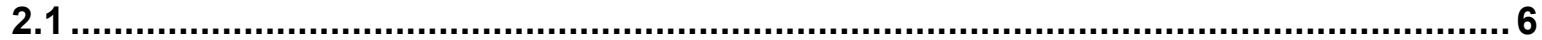

3 Überblick über wichtige Standards und Mindestanforderungen - die 9

Prinzipien ......................................................................................................

3.1 Grundsatz 1 - Datenqualität und Harmonisierung der Datensammlung .......... 11

3.2 Grundsatz 2 - Aufnahme von vergleichbaren Unternehmen und/oder

Privatpersonen in die Kontrollgruppe.

3.2.1 Auswahl von Kontrollgruppen durch die so genannte "Propensity Score Nearest

Neighbour Matching Methode" .......................................................................... 14

3.3 Grundsatz 3 - Verwendung der difference-in-difference Methode und

balancierte Paneldaten ......................................................................................15

3.3.1 Die difference-in-difference Methode ......................................................... 16

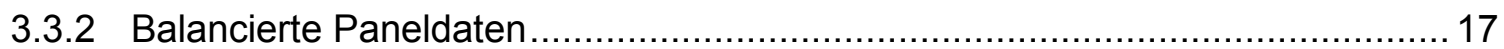

3.3.3 Standards für die Berechnung von ökonomischen Effekten ............................ 17

3.4 Grundsatz 4 - Behandlung von Außenseitern............................................... 18

3.5 Grundsatz 5 - Langzeit Serien...................................................................... 18

3.6 Grundsatz 6 - Robustheits Test ................................................................. 18

3.7 Grundsatz 7 - Effektindikatoren sollten relativiert werden ............................... 18

3.8 Grundsatz 8 - Peer Review der Resultate...................................................... 19

3.9 Grundsatz 9 - Fehler und Stress Tests..................................................... 19

4 PART II - Standard for performance objectives: Key performance indicators 19

4.1 Ex ante Messung 20

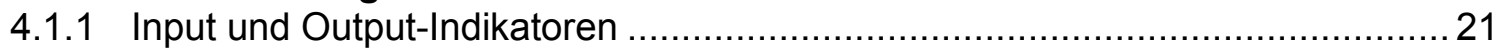

4.2 Basismaßnahmen für ex post Messungen ……………………………….... 21

4.2.1 Outcome und Impact Indikatoren ............................................................ 23

5 TEIL III - Übersicht über die Leistungskennzahl, Effektmessung und

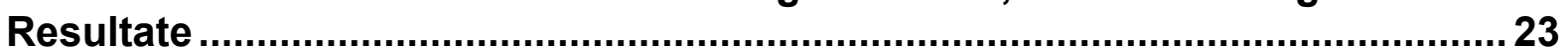

5.1 Resultate von Effektmessungen in Dänemark ................................................. 23 
Exzellente Methoden für das Messen des Effekts von politischen Innovationen für Forschung und Innovation ist der Schlüssel für evidenzbasierte politische Entscheidungsfindung. Der Bereich der quantitativen Effekt Messung (quantitative effect assessment) befindet sich in ständiger Entwicklung. 2011 hat das Ministerium für Forschung und weiterführende Bildung das erste Handbuch für exzellente ökonometrische Messung herausgebracht": "Central Innovation Manual on Excellent Econometric Evaluation of the Impact of Interventions on $R \& D$ and Innovation in Business (CIM)". Auf Grund neuer Erfahrungen in der Forschung, neuer Effektauswertung, verbesserter Datensammlung und längerer Zeitrahmen ist es an der Zeit für ein 2.0 Update. CIM 2.0 schafft einen Rahmen für sowohl ein Minimum an Analyse- und Qualitätsstandards für Datenmaterial als auch Standards für Key Performance Indikatoren (KPI) für ökonometrische Effektstudien öffentlicher Forschung, von Innovationsprogrammen und politischen Initiativen. Des Weiteren bietet CIM 2.0 sowohl einen Überblick über die wichtigsten KPIs und Zielvorgaben als auch über die ökonometrische Effektmessung des dänischen Forschungs- und Innovationssystems.

Warum CIM?

Entscheidungsträger und Programmmanager suchen ständig nach den besten und beständigsten Methoden für die Identifizierung und Verifizierung des Effektes von politischen Initiativen. Das CIM versteht sich als Handbuch für exzellente Methoden für das Durchführen von sowohl ökonomischen als auch nicht-ökonomischen ökonometrischen Effektmessungen.

Struktur des Handbuchs

Der Report wird in drei Teile gegliedert. Teil I beinhaltet ein Handbuch für exzellente ökonometrische Messung des Effekts von Interventionen für Forschung und Entwicklung (FuE) und Innovation im Bereich Wirtschaft, Teil II enthält Standards für KPIs und Teil III präsentiert einen Überblick über ökonometrische Effektmessung dänischer Forschungs- und Innovationspolitik.

Teil I: Handbuch für exzellente ökonometrische Effektmessung von Interventionen in FuE und Innovation im Bereich Wirtschaft

Neun Grundsätze, welche wichtige Standards und Minimalanforderungen für exzellente ökonometrische Effektmessung darstellen, werden anhand von Erfahrungen mit Effektmessungen modernster dänischer FuE und Innovationsprogrammen formuliert.

Teil II: Standards für Key Performance Indikatoren (KPIs)

Teil II verschafft einen Überblick über ein umfassendes Set an Input-, Output-, Outcome und Effektperformance Indikatoren zum Erfassen des Effekts von Forschungs- und Innovationsprogrammen,. Obwohl CIM 2.0 Standards für Effektmessungen vorgibt, sollte Raum für Flexibilität bleiben.

\footnotetext{
${ }^{1}$ http://fivu.dk/en/publications/2012/central-innovation-manual-on-excellent-econometric-impact-analyses-ofinnovation-policy-cim?searchterm $=\mathrm{CIM}$
} 
Part III: Überblick über die wichtigsten Key Performance Indikatoren, Effektmessungen und Resultate Dänemarks

Dieser Teil verschafft einen Überblick über die wichtigsten ökonometrischen Effektmessungen der dänischen Forschungs- und Innovationsprogramme, FuE Systeme und deren Effekte. Teil III beinhaltet auch einen Überblick über die gängigsten Key Performance Indikatoren, die in den Studien dänischer Forschungs- und Innovationsprogramme Anwendung finden.

\subsection{Danksagung}

Dieses Handbuch wurde in enger Zusammenarbeit mit universitären und privaten Forschern entwickelt. Des Weiteren wurde es bei Workshops und Seminaren zwischen 2010 und 2013 an der Copenhagen Business School (CBS) und im Ministerium für Forschung und weiterführende Bildung diskutiert und es sind wertvolle Kommentare von mehreren dänischen Ministerien, darunter dem Finanzministerium, dem Ministerium für Wirtschaft und Wachstum, dem Ministerium für Klima und Energie, dem Ministerium für Lebensmittel, Landwirtschaft und Fischerei, dem Außenministerium und dem Ministerium für Umwelt darin enthalten. Spezieller Dank geht an Professor Anders Sørensen und Dr. Johan Kuhn von der Copenhagen Business School (CBS) und an Michael Mark von der DAMVAD Consulting A/S.

Jahrzehntelang haben Entscheidungsträger und Programmmanager international an der Überwachung und Messung von Forschungs- und Innovationsprogrammen gearbeitet. Gründliche Messung des Effekts von Innovationspolitik ist der Schlüssel für beweisbasierte politische Entscheidungsfindung ${ }^{2}$. Auf Grund vieler wirtschaftlicher Herausforderungen, internationalem Wettbewerbs und dem Streben nach der Führungsposition im Bereich FuE und Innovation, verlangen Entscheidungsträger Effekt und Rechenschaft von Forschungs-, Entwicklungs- und Innovationsprogrammen und politischen Initiativen. Effektmessung kann dabei eine stabile und zuverlässliche Beweisgrundlage für die Performance politischer Initiativen bilden und festlegen, ob ein bestimmtes Programm die gewünschten Resultate und Effekte erzielen konnte.
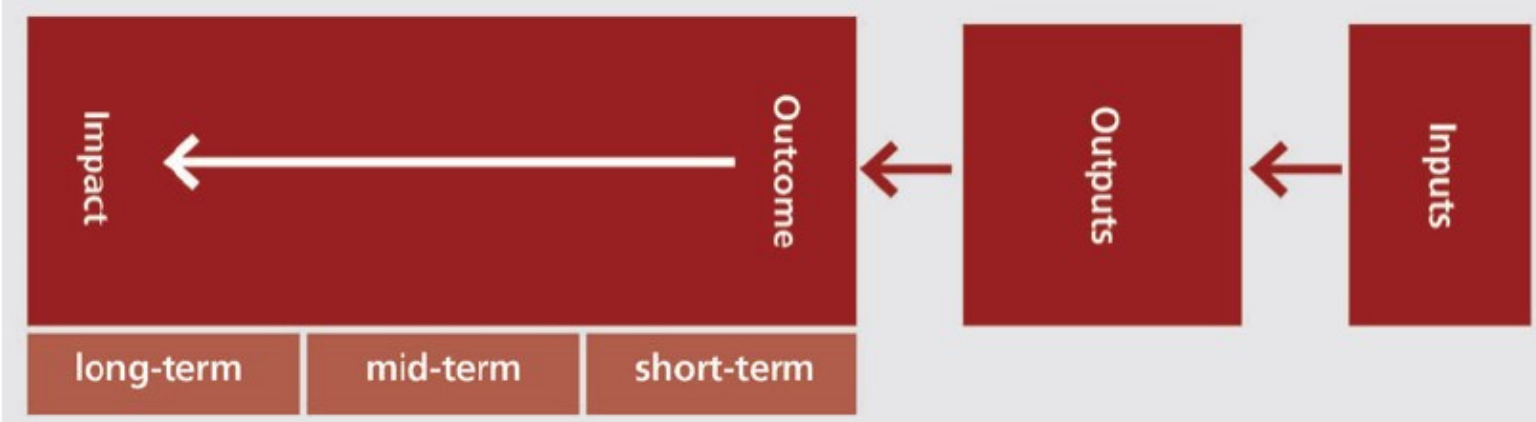

Effektmessungen sind Teil eines breiteren Programms von beweisinduzierter politischer Entscheidungsfindung. Dieser wachsende globale Trend ist gekennzeichnet durch den Wechsel des Fokus von Inputs (Investition) und Outputs (Aktivitäten) hin zu Outcomes (Resultate) und Impacts (Effekt).

\footnotetext{
${ }^{2}$ Communication from the Commission to the European Parliament, the Council, The European Economic and Social Committee and the Committee of the Regions: "Measuring innovation output in Europe: towards a new indicator", final 624, 13 September 2013
} 
Dieser steigende Fokus wurde, unter anderem, durch die $\mathrm{OECD}^{3}$ gefördert, welche diesem Gebiet große Aufmerksam geschenkt hat durch gemeinsames Bestreben von einer Mehrzahl der $27 \mathrm{EU}$ Ländern, Korea, Norwegen, Schweiz, Russland, Türkei, Süd Afrika und der Mehrheit der südamerikanischen Länder.

Die Vision der dänischen Agentur für Wissenschaft, Technologie und Innovation ${ }^{4}$ ist es, die ökonometrische Effektmessung von öffentlichen dänischen Forschungs- und Innovationsprogrammen in den kommenden Jahrzehnten international als best practice zu etablieren. ${ }^{5}$ Die Aufgabe von CIM 2.0 ist es Minimalanforderungen und Standards für Methoden und Daten, die für die Implementierung von exzellenten ökonometrischen Effektmessungen von Innovations- und Forschungspolitik notwendig sind, zu errichten.

Was ist das CIM 2.0?

Das Handbuch für Exzellente Ökonometrische Effektmessung Öffentlicher Investition in Forschung und

Entwicklung (CIM) legt Fokus auf Methoden ökonometrische Resultat- und Effektmessung bei der

Analyse von Investitionen in den Bereichen der öffentlichen und privaten Forschung und Entwicklung

(FuE), Unterricht und Innovation.

CIM 2.0 ist das Resultat der Messungsstrategie der dänischen Agentur für Wissenschaft, Technologie und Innovation, welche als 6-jahres Forschungs- und Innovationsprojekt über die Messung der Performance im Bereich Innovation errichtet wurde. Die Hauptelemente des 6-jahres-Projekts sind die mehr als 20 Messungen (siehe Kapitel 4.1), die zwischen 2007 und 2012 durchgeführt wurden. Das Forschungsprojekt wurde bis 2014 verlängert und umfasst nun mehr als 25 ökonometrische Impact Studien und Messungen.

\section{Text Box 1. Definition von Effektmessung}

Effektmessungen sind Evaluierungen, welche auf die Beantwortung von Ursache-Effekt (cause-and-effect) Fragen abzielen. Im Gegensatz zu allgemeinen Evaluierungen, welche viele Arten von Fragen beantworten können, zielen Effektmessungen auf eine bestimmte Frage ab: Was ist der Effekt (oder der kausale Effekt) eines Programms oder einer politischen Initiative? Diese grundlegende Frage beinhaltet eine wichtige kausale Dimension: Das Interesse liegt ausschließlich auf dem isolierten Effekt des Programmes oder politischen Initiative, d.h. dem Effekt von Resultaten (Outcomes), welcher das Programm oder die Richtlinie direkt bedingt. Die grundlegende Form der Effektmessung wird die Effektivität einer gegebenen Richtlinie oder Programms testen. Qualitative Daten, Kontrolldaten und Evaluierungen werden benötigt um die Implementierung von Programmen und Politischen Initiativen zu verfolgen und um jene Fragen über Fortschritt zu prüfen, denen hinsichtlich Aufklärung und Interpretation der Ergebnisse von Effektmessungen besondere Bedeutung zukommt. Aus diesem Grund sind Effektmessungen und andere Formen von Evaluierung eher Gegenstücke als Ergänzungen.

\footnotetext{
${ }^{3}$ OECD (2008), Science, Technology and Industry Outlook.

${ }^{4}$ Im Ministerium für Forschung und weiterführende Bildung: http://fivu.dk/en?set language=en\&cl=en

${ }^{5}$ In den Reporten 'Clusters Are Individuals - Benchmarking Insights from Cluster Management Organizations and Cluster Programs' der Kompetenznetze Deutschland (VDI/VDE Innovation + Technik), 2011, und 'Service innovation: Impact analysis and assessment indicators' der Europäischen Kommission Pro-Inno Net EPISIS, 2011, die Messung der ökonometrischen Performance des dänischen Ministeriums für Forschung und weiterführende Bildung werden hervorgehoben als international best practice.
} 
CIM 2.0 wurde in Übereinstimmung mit den neuesten und besten ökonometrischen Forschungsmethoden implementiert und besitzt darauf basierend eine so hohe forschungsbezogene Qualität, welche Publikationen von Methoden und Ergebnissen in den höchst angesehenen internationalen Fachzeitschriften in den relevanten Gebieten ermöglicht.

Primär zielt der CIM 2.0 auf Programmmanager und politische Entscheidungsträger in den dänischen Ministerien, Regierungsbehörden und Regionen ab, die von Programmen und Politik für Forschung und Innovation berührt sind. Weitere Zielgruppen sind externe Interessenvertreter, Messungsexperten (von Organisationen, Regionen, Forschung, Technologie, Unterrichts- und Wissenseinrichtungen, etc.), Forscher, Gewerkschaften und Handelsverbände, die an einem Dialog mit dem Ministerium für Forschung und weiterführende Bildung über die Durchführung von Messungsstudien und das Dokumentieren der Effekte der Innovationspolitik Interesse haben -

mit anderen Worten, alle Ministerien und Behörden, die an ökonometrischer Effektmessung interessiert sind.

CIM 2.0 ist nicht identisch mit der Arbeit in anderen Ländern ${ }^{6}$, da die Hauptaufgabe darin besteht, ein eindeutiges Set an minimalen Anforderungen für die Führung von sogenannten exzellenten ökonometrischen Effektmessungen von Innovationspolitik zu etablieren. CIM 2.0 bildet den Rahmen für ein Standard- Effektmessungsprogramm, welches es ermöglicht, exzellente Effektmessungen von Interventionen für Forschungs- und Innovationsprogramme im Bereich der Wirtschaft durchzuführen, um den Effekt unterschiedlicher Programme vergleichen zu können. CIM 2.0 ist folglich kein Versuch ein Handbuch für eine Vielzahl von Methoden darüber darzustellen, wie der weitläufige Effekt von Forschungs- und Innovationsprogrammen auf die Wirtschaft abzuschätzen ist.

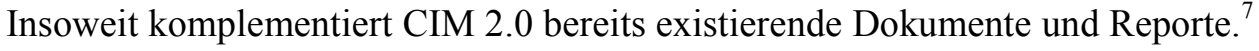

Obwohl CIM 2.0 Anforderungen und Standards für Effektmessungen angibt, wurde zusätzlich berücksichtigt, Raum für Flexibilität und Diversität zu gewähren. Des Weiteren ist es der Zweck dieses Handbuchs zur internationalen Diskussion und dem vorhandenen Wissen über exzellente Methoden für Messungen von Performance in Forschungs-, Innovations- und Wirtschaftspolitik beizutragen.

Da exzellente Effektmessungen die Arbeit ausgezeichneter Forscher und Gutachter, die Finanzierung von Forschungs- und Messungsprojekten und einen nicht limitierten Zugang zu relevanten Qualitätsdaten von öffentlichen Programmen und politischen Initiativen voraussetzt, muss eine enge Zusammenarbeit zwischen Forschern und Gutachtern auf der einen Seite und Unternehmern, Programmmanager und politischen Entscheidungsträgern auf der anderen Seite etabliert werden. Exzellente ökonometrische Effektmessungen von öffentlichen Programmen und politischen Initiativen müssen auf gemeinsamen Respekt aufbauen.

Der Erfolgsfaktor einer exzellenten Effektmessung hängt auch davon ab, in welchem Ausmaß die Messung auf ein relevantes und limitiertes Set von Effekt-Variablen abzielt und in welchem Grad es hinsichtlich von Methoden maßgeschneidert ist. Des Weiteren hängt die Relevanz und das Ergebnis einer Messung vom Zusammenhang zwischen den Messungsvorgängen und den Anforderungen des Sponsors ab. In diesem Zusammenhang können eine enge Bindung und ein hohes Level an Kooperation zwischen den Programmsponsoren und Gutachtern zur Formulierung und Durchführung

\footnotetext{
${ }^{6}$ Siehe Guidance on evaluating the impact of interventions on business, Department for Business, Innovation and Skills (BIS), August 2011

${ }^{7}$ Z.B. The role of evaluation in evidence-based decision-making, Department for Business, Innovation and Skills (BIS), August 2010, and The Green Book - Appraisal and Evaluation in Central Government, Treasury Guidance, London, United Kingdom, and The Magenta Book: guidance notes for policy evaluation and analysis, Government Social Research Unit, HM Treasury, London, United Kingdom (October 2007)
} 
einer effektiven und zweckmäßigen (d.h., dass die Studie zum politischen Verständnis beiträgt) Messung führen.

Diese Ansicht wird von einer großen europäischen Studie über Messungspraxis und Methoden in den europäischen Ländern geteilt. Dies wurde von Technolopolis in einer Messung der ökonometrischen Effektstudie des Dänischen Innovations-Konsortium-Programms (siehe Textbox weiter unten) hervorgehoben, welche als Beispiel für die Art von exzellenten ökonometrischen Effektmessungen von FuE und Innovationspolitik angeführt werden.

\section{Messungssynopsis von Technolopolis über: "An Analysis of Firm Growth Effects of the Danish Innovation Consortium Scheme"}

Interviewer und Author der Fallstudie: Paul Cunningham, Manchester Institute of Innovation Research

\section{Einleitende Information}

Das Dänische Innovations-Konsortium-Programm ist ein nationaler Finanzierungsplan unter der Leitung des dänischen Rates für Technologie und Innovation (Danish Council for Technology and Innovation, RTI) in Kooperation mit der Dänischen Agentur für Wissenschaft, Technologie und Innovation (Danish Agency for Science, Technology and Innovation, DASTI). Die ökonometrische Effektmessung dieses Programms trägt den Titel "An Analysis of Firm Growth Effects of the Danish Innovation Consortium Scheme", welches eine provisorische Messung der Dänischen Agentur für Wissenschaft, Technologie und Innovation darstellt. Die Messung wurde zwischen September 2009 und April 2010 von J.M. Kuhn vom Centre for Economic and Business Research, Copenhagen Business School (CBS), durchgeführt. Das Budget für die Messung betrug zirka €40,200 mit dem Ziel, den ökonomischen Effekt zu untersuchen. In einer counter-factual Analyse, wurden 220 Unternehmen untersucht, die mindestens an einem Innovationskonsortium, welches ein Unternehmensregister-Datenset verwendet, teilgenommen hatten. Der Fokus lag dabei auf Entwicklungen der Unternehmensebene in zwei Erfolgsparametern: Bruttogewinne und Beschäftigung. Innovationskonsortia finanzieren und ermöglichen eine Kooperation zwischen privaten Unternehmen und Forschungs- und Wissensinstituten und tragen dadurch zur Weitergabe von Wissen und Forschungsresultaten vom Forschungsin den Wirtschaftssektor bei.

\section{Zusammenfassung}

Ziele der evaluierten Initiative waren folgende: Durch das Installieren von Innovationskonsortia (bestehend zumindest aus zwei Unternehmen, einer Forschungseinrichtung und einer Organisation für die Weitergabe von Wissen) sollen gemeinsame Projekte beworben werden, die das Entwickeln und das Ausreifen forschungsbasiertem Wissens zum Nutzen dänischer Unternehmen und der dänischen Handelsgemeinschaft bezwecken sollten - besonders KMUs. Ziele und Hauptfragen der Messung waren: Bestimmung des wirtschaftlichen Effekts der Initiative (hinsichtlich Schaffen von Arbeitsplätzen und Bruttogewinn) und die Überlebensrate von am Programm teilnehmenden Unternehmen im Vergleich zu nicht-teilnehmenden Unternehmen.

Die Messungsmethodik war der Gipfel einer spezifischen Herangehensweise an das Forschungsprojekt unterstützt von der kommissionären Agentur und dem Gutachter. Die Herangehensweise und Hauptforschungsmethode umfasste einen Kontrollgruppenvergleich von spezifischen Wachstumsfaktoren (nämlich Bruttogewinn und Beschäftigung). Die Hauptbezugsquellen erfassten Information gewonnen aus den Programm-Kontrolldaten (der teilnehmenden Unternehmen), früheren Messungen (von 2007 und 2008), Unternehmensfinanzreporten (eines auswärtigen öffentlichen Datensets) und Fusionierungs- und Übernahmedaten (ebenfalls öffentlich).

Die Hauptergebnisse der Studie zeigten, dass die am Programm des Innovationskonsortiums teilnehmenden Unternehmen signifikante Zunahme an Bruttogewinn und Beschäftigung im Vergleich zum Wachstum vor der Teilnahme und zu den Wachstumsentwicklungen von Unternehmen in der Kontrollgruppe verzeichnen konnten. Dennoch war der Effekt deutlicher bei kleineren Unternehmen (Bruttogewinn $<\$ 20$ m oder $<150$ Mitarbeiter) als bei großen, teilnehmenden Unternehmen. Bezüglich Überlebensraten konnten keine Unterschiede ermittelt werden. Hinsichtlich der Lehren von der Messung von Unterstützungsmaßnahmen für Innovationen, wurde der (relativ einfache und limitierte) Zugang der Analyse für diesen Typ von Programm als bevorzugt angesehen, obwohl regelmäßige Updates der Daten zu zusätzlicher Qualität und Verfeinerung beitragen könnten - z.B. zur Identifizierung jener Unternehmen, die am meisten profitieren oder welche Innovationskonsortia besser arbeiten als andere. Es sollte erwähnt werden, dass andere Eigenschaften der Maßnahme (Management- und Prozessangelegenheiten, Teilnehmerinteraktion) auch durch gezielte, separate Messungen beurteilt wurden. 


\section{Konklusion und gewonnene Erkenntnisse}

Die Messung war erfolgreich hinsichtlich Entwicklung einer Methodik, die höchst übereinstimmend mit den Zielen der in Auftrag gebenden Behörde (Dänische Agentur für Wissenschaft, Technologie und Innovation), welches sich an einen begrenzten Fragenkatalog richtet, nämlich die Abschätzung des ökonomischen Effekts des IC Programms auf teilnehmende Unternehmen. Dieses Niveau an Beständigkeit wurde erreicht durch die enge Verbindung und ein Maß an Interaktion zwischen den Gutachtern und Unternehmern und durch die Möglichkeit der Anwendung der Methodik in zwei separaten Messungen, welche die weitere Verfeinerung der Herangehensweisen zulassen. Der persönliche Einsatz und das große Interesse der Hauptsponsoren (Direktor des Centers für strategisches Forschung und Wachstum innerhalb der Dänischen Agentur für Wissenschaft, Technologie und Innovation) kann auch als Hauptfaktor für den Erfolg der Methodik hervorgehoben werden. Insgesamt wurde der gesamte Prozess als "besonders angenehm" von den Gutachtern bezeichnet.

In Bezug auf die Herangehensweise wurde vom Programmmanager erwähnt, dass die Messung ein hohes Maß an Nützlichkeit erzielen konnte: Es wurden essentielle Antworten auf spezifische Fragen gegeben, welche für politische Rechtfertigung und budgetäre Anforderungen benötigt waren und es wurde ein eindeutiger, quantitativen Beweis für den Erfolg des Programmes geliefert. Zusätzlich hat die Messung zur Entwicklung einer sowohl reproduzierbaren und übertragbaren Methodik geführt. Wie erwähnt war die Messung stark abhängig von hochqualitativen, umfassenden Daten sowohl der teilnehmenden Unternehmen als auch von den Unternehmen der Kontrollgruppe, die für die kontrafaktische Analyse benötigt wurden. Jedoch stellt die Anforderung an solch hochqualitative Information einen potentiell limitierenden Faktor für das Adoptieren der Herangehensweise in anderen Zusammenhängen dar. In Abwesenheit existierender Datenquellen wäre es erforderlich mehrere Messungsressourcen zu erweitern, um die notwendigen Daten generieren zu können. Ferner würden sie nicht regelmäßig upgedatet wie im Falle Dänemarks. Jedoch sollte erwähnt werden, dass laut Gutachter der Gebrauch der Methoden auch als potentielle Methodik für die Messung der Eureka Programmteilnahme erforscht wurde. Zusätzlich soll hervorgehoben werden, dass die gleiche Herangehensweise in anderen dänischen Innovationsförderungsprogrammen, wie z.B. dem Industrie-Doktorat Programm, internationalen Kooperationsprojekten und dem Innovations-Assistenzprogramm, angewendet wurde.

Für die Bestimmung der Unternehmen der Kontrollgruppe wurde ein sorgfältiger Auswahlprozess vorgesehen, der gewährleisten konnte, dass diese den am Programm teilnehmenden Unternehmen in Hinblick auf die Charakteristika vor der Beteiligung am Programm sehr ähnlich waren. Dies konnte (wenngleich nicht zur Gänze) die Wahrscheinlichkeit, dass äußere Charakteristika für beobachtete Abweichungen in den Variablen für Wirtschaftswachstum (economic growth variables) zwischen den zwei Gruppen als Folge von der Teilnahme am Programm verantwortlich waren, reduzieren. Da die Methodik der Messung relativ unkompliziert war, wäre ihr Gebrauch von anderen Gutachtern nicht von der Notwendigkeit fortgeschrittener oder spezieller Kompetenzen abhängig. Bei der Aufnahme hat die Messung nicht, wie sonst üblich signifikant für diese Art von Innovationsförderungsmaßnahme, auf Aspekte abgezielt, wie z.B. Zufriedenheit der Teilnehmer, Aufnahmestatistiken und Argumentation für die Teilnahme, Management und administrative Prozessangelegenheiten, Aspekte von Wissensübertragung, Effekte auf andere an IC teilnehmende Partner, Aktivitäten (Outputs) (z.B. Publikationen, Patente) und damit verbundene Qualitätsprobleme, etc. Der Unternehmer schätzte, dass sie nur etwa $20 \%$ der gesamten Information, die für die vollständige Messung des IC Programmes benötigt war, lieferte; jedoch war dies zur Gänze bekannt. Aus diesem Grund wurden separate, begleitende Messungen durchgeführt unter Verwendung verschiedener Gutachter über verschiedene Zeitrahmen unter diesen spezifischen Aspekten.

Diese Herangehensweise bringt insoweit breite Erkenntnisse für die Messung von Programmen, als sie das Durchführen von separaten, mehr zielgerichteten Messungsstudien, die von verschiedenen Gutachtern mit individuellen Fähigkeiten und Expertisen im Gegensatz zu einer einzigen, einmaligen, mehr umfassenden Messung unternommen wurden, ermöglicht. Dies ist besonders dienlich in jenen Fällen, in denen Angelegenheiten hinsichtlich Timing wichtig sind, da dies Kompromisse zwischen der Durchführung einer Messung in der frühen Phase für die Gewinnung von Management-/Prozessinformation, die die eventuelle Notwendigkeit für einen Richtungswechsel der Administration des Programmes vorschlagen könnten, und einer Halbzeitmessung, in der aufkommende Networkingeffekte und Wissenstransfers-/Wissensoutputeffekte bewertet werden, und schließlich einer Langzeitmessung, in welcher verschiedene Aspekte von Effekten bewertet werden, vermeidet.

Zusammenfassend sind die Hauptschlüsse folgende:

- Die Messung war hoch fokussiert auf ein begrenztes Set von Effekt-Variablen und demnach gänzlich maßgeschneidert (hinsichtlich Methoden und zeitlicher Planung), um die Programmeffekte in diesem Zusammenhang zu erörtern. 
- Der Erfolg der Messung kann zum Teil der Konsistenz zwischen den Herangehensweisen an die Messung und den Anforderungen des Sponsors/der Sponsorin des Programms angerechnet werden.

- Die enge Verbindung und das hohe Maß an Interaktion zwischen Programmsponsoren und Gutachtern führte zur Formulierung und Implementierung einer effektiven und nützlichen Messung (nützlich im Sinne des Beitragens der Studie zum politischen Verständnis).

- Der Erfolg der ursprünglichen Studienmethodik beruht auf der Existenz und Verfügbarkeit umfassender und robuster Daten auf Unternehmensebene.

- Die Studie war in der Lage diese Daten dazu zu verwenden, um verlässliche Vergleichsdatensets von teilnehmenden und nicht-teilnehmenden Unternehmen zu produzieren.

- Unter den oben angeführten Voraussetzungen, scheint die Methodik einen guten Rahmen für ihre Verwendung in weiteren ähnlichen Modellen zu bieten.

- Der breitere Messungszugang, welcher variable Zeitplanung und dazu passende Methodik verwendet, schafft

Erkenntnisse für zukünftige Messungen.

Weitere Information unter: http://fivu.dk/en/publications/2010/an-analysis-of-firm-growth-effects-of-the-danishinnovation-consortium-scheme

3 Überblick über wichtige Standards und Mindestanforderungen - die 9 Prinzipien

Die größte Herausforderung in der Durchführung einer effektiven Effektmessung ist die Identifizierung der kausalen Verbindung zwischen Projekt, Programm und KPIs. Demnach sind die Anforderungen des CIM 2.0 auf exzellente ökonometrische Effektmessungen (1) hohe Datenqualität, (2) Anwendung der neuesten forschungsbasierten statistischen Methoden (3) und eine hohe Qualität an Kontrollgruppen. Auf dieser Basis sind im CIM 2.0 politische Initiativen in Form von neun Prinzipien als Mindestanforderungen an exzellenten Effektmessungen vorausgesetzt.

\section{Prinzipien: Mindestanforderungen an exzellente ökonometrische Effektmessungen}

\begin{tabular}{|c|c|}
\hline \multirow[t]{2}{*}{ Daten } & 1. Datenqualität und Harmonisierung der Datensammlung \\
\hline & $\begin{array}{l}\text { Etablierung von Standards für Datensammlung, inklusive Standards für Input-Daten und } \\
\text { Registrierung in Datenbanken für jedes Forschungs- und Innovationsprogramm. } \\
\text { - Standards für Datensammlung sind harmonisiert quer durch alle Forschungs- } \\
\text { und Innovationsprogramme in der Datenbank der Dänischen Agentur für } \\
\text { Wissenschaft, Technologie und Innovation (die Innovation Denmark } \\
\text { Datenbank). } \\
\text { - Input-Daten sollen dabei von höchster Qualität sein, damit sie mit nationalen } \\
\text { und internationalen Datenbanken verlinkt werden können. }\end{array}$ \\
\hline \multirow[t]{4}{*}{ Design } & $\begin{array}{l}\text { 2. Auswahl vergleichbarer Unternehmen und/oder Einzelpersonen als } \\
\text { Kontrollgruppe }\end{array}$ \\
\hline & $\begin{array}{l}\text { Die Auswahl von vergleichbaren Unternehmen und/oder Einzelpersonen für } \\
\text { Kontrollgruppen muss auf der Abstimmung von so vielen als relevant beobachteten } \\
\text { Charakteristika wie möglich basieren. Die höchste Qualität und beste Interpretation der } \\
\text { Daten muss Voraussetzung sein. }\end{array}$ \\
\hline & $\begin{array}{l}\text { Auswahl der Kontrollgruppen: } \\
\text { - Verwendung von propensity score und der nearest neighbour matching } \\
\text { Methode für die Auswahl der am besten vergleichbaren Kontrollgruppe } \\
\text { - Alternative Methoden wie Lotterie und Reihung könnten auch erwägt werden, } \\
\text { z.B. in Robustheit-Tests (siehe Prinzip 6) }\end{array}$ \\
\hline & 3. Difference-in-difference Methode \\
\hline
\end{tabular}




\begin{tabular}{|c|c|}
\hline & Verwendung der difference-in-difference Methode und ausgewogene Panel-Daten. \\
\hline & 4. Behandlung von Ausreißern \\
\hline & $\begin{array}{l}\text { Ausreißer müssen nach den etablierten internationalen Methoden im Bereich der } \\
\text { ökonomischen Forschung und ökonometrischen Methoden behandelt werden. }\end{array}$ \\
\hline & 5. Langzeit Serien \\
\hline & $\begin{array}{l}\text { Gewährleistung von hoher Datenqualität durch Langzeitserien von mindestens 6-15 } \\
\text { Jahren mit minimalen Datenlücken. Sowohl nationale Statistiken von } \\
\text { Unternehmensdaten und persönlichen Daten als auch DASTIS' Innovation Denmark } \\
\text { Database für Forschungs- und Innovationsprogramme müssen je nach Instrument mit } \\
\text { Zeitserien von 20-25 Jahren angewendet werden. }\end{array}$ \\
\hline & 6. Robustheit-Test \\
\hline & $\begin{array}{l}\text { Robustheit-Tests werden für Studien mit langen Zeitserien und vielen Observationen } \\
\text { empfohlen. Im Falle von Datenlimitierung, z.B. limitierten Zeitserien und } \\
\text { Beobachtungen, ist es Voraussetzung Effektmessungen unter Anwendung von Methoden } \\
\text { durchzuführen, die die Robustheit der Resultate testen. }\end{array}$ \\
\hline & 7. Relative Effekt-Indikatoren \\
\hline $\begin{array}{l}\text { von } \\
\text { Resultaten }\end{array}$ & $\begin{array}{l}\text { Die KPIs sollten mit relativen Zahlen angegeben werden, um den Vergleich von } \\
\text { ungeraden Einheiten zu vermeiden, z.B. durch Unterschiede in Wachstumsraten oder } \\
\text { anderen relevanten Verhältniszahlen. }\end{array}$ \\
\hline & 8. Peer-Review von Resultaten \\
\hline & $\begin{array}{l}\text { Die Qualität und Aussagekraft von Effektmessungen müssen mit unabhängigen } \\
\text { Forschungsorganisationen diskutiert werden, die nicht an der Analyse teilgenommen } \\
\text { haben, z.B. durch Peer-Reviews, Forschungsseminare, Workshops mit politischen } \\
\text { Entscheidungsträgern, etc. Es ist wünschenswert, dass die Resultate der } \\
\text { Effektmessungen für die Publizierung in anerkannten Fachzeitschriften geeignet sind. }\end{array}$ \\
\hline $\begin{array}{l}\text { Kritische } \\
\text { Kernpunkte }\end{array}$ & $\begin{array}{l}\text { 9. Fehler und Stress-Tests } \\
\text { Effektstudien tragen auch zum politischen Lernen bei. Politische Entscheidungsträger } \\
\text { und Programmmanager sollten kritische Kernpunkte, fehlerhafte Resultate, Scheitern } \\
\text { von Projekten und Programmen, etc. als Teil der Messung akzeptieren. Der } \\
\text { Auftraggeber/die Auftraggeberin der Messung sollte von Fehlern lernen und nicht nur } \\
\text { auf Erfolgsgeschichten aus sein. Eine enge Beziehung und ein hohes Level an } \\
\text { Interaktion und gemeinsamen Vertrauens zwischen Programmsponsoren und Gutachtern } \\
\text { steigern die Wahrscheinlichkeit der Formulierung und Umsetzung einer effektiven und } \\
\text { brauchbaren Messung, brauchbar in dem Sinn, dass die Studie zum politischen Lernen } \\
\text { und neuem Wissen über die Stärken und Schwächen des Programmes beigetragen hat. }\end{array}$ \\
\hline
\end{tabular}




\subsection{Grundsatz 1 - Datenqualität und Harmonisierung der Datensammlung}

Gute Datenqualität ist für die Messung des Effekts von Forschung und Innovation von absoluter Notwendigkeit. Als Minimum sollten Input-Daten etwas über den Beginn eines Programms oder einer politischen Initiative und über die Teilnehmer aussagen können. Input-Daten werden von Programmmanagern gesammelt, oft im Zeitpunkt des Erbringens der Dienstleistung als Teil der Routine. Sowohl zugelassene als auch abgewiesene Projekte sollten systematisch registriert werden. Daten von abgewiesenen Projekten können für die Auswahl von Kontrollgruppen nützlich sein. Als Standard sollten die folgenden Input-Daten für alle Forschungs- und Innovationsprogramme gesammelt werden:

- Variable für jedes Projekt: Name des Programms, Titel des Projekts, Status über die Zu- oder Absage von Förderungen, Bewerbungsjahr, Datum des Projektbeginns, Datum für das Ende des Projekts, Auflistung des Gesamtbudgets und aller Förderungen

- Variable jedes teilnehmenden Projektpartners: Firmenkennzahl, Name, Postleitzahl, Region, Zahl der Angestellten, Industrie Bereich (NACE-Klasse).

- Variable aller teilnehmenden Privatpersonen an einem Projekt: Sozialversicherungsnummer, Name, Geschlecht, Alter, Ausbildung.

Die Eingabedaten eines jeden Forschungs- und Innovationsprogramms der Dänischen Agentur für Wissenschaft, Technologie und Innovation sind harmonisiert und Teil der Innovation-Denmark Datenbank.

Des Weiteren wird das Sammeln von Eingabedaten in das Design jedes neuen Forschungs- und Innovationsprogramms inkludiert und nicht erst dann, wenn ein Programm gemessen werden soll.

Eingabedaten reichen für die Effektmessungen nicht aus, dadurch werden nationale und/oder internationale Statistiken angewendet. Folgende nationale Statistiken werden in Verbindung mit Effektmessungen verwendet:

- FuE Statistiken (Statistics Denmark)

- Buchhaltungs-Statistiken (Statistics Denmark)

- Community Innovation Survey (CIS) (Statistics Denmark)

- Bildungs-Statistiken (Statistics Denmark)

- Patent-Statistiken (Statistik Denmark)

- Arbeitsmarkt-Statistiken (Statistics Denmark)

- Gehalts-Statistiken (Statistics Denmark)

- Zentraler Wirtschaftsregister Dänischer Handels- und Unternehmensagenturen / Købmandsstandens Oplysningsbureau/Experian A/S (Danish Business World's Information Agency)

Firmenkennzahl oder Sozialversicherungsnummer der Teilnehmer bilden die wichtigsten Eingabedaten, da es sich dabei um die Verknüpfung von Eingabedaten, die für jedes Programm gesammelt wurden, und den nationalen Statistiken handelt. Es ist wichtig, dass zumindest das Sortieren der Beobachtung und das Reinigen von Daten in zu vergleichenden Forschungs- und Innovationsprogrammen auf gleiche Art und Weise vollzogen werden ${ }^{8}$.

\footnotetext{
${ }^{8}$ Als Beispiel können herkömmliche Doktorate und Industrie-Doktorate verglichen werden, siehe DASTI/FI(01/2011), und die Teilnahme von Unternehmen an EUREKA Projekten und Innovationskonsortia, siehe DASTI/FI(15/2011).
} 
3.2 Grundsatz 2 - Aufnahme von vergleichbaren Unternehmen und/oder Privatpersonen in die Kontrollgruppe

Der Effekt der Teilnahme an einem Forschungs- und Innovationsprogramm erfolgt zumeist indirekt und ist dadurch schwer zu messen und zu identifizieren. Es ist schwierig den eigentlichen Effekt, der das Resultat vieler und variierender Faktoren sein kann, zu isolieren. Des Weiteren ist es schwierig die Kausalität der Teilnahme an einem Forschungs- und Innovationsprogramm zu identifizieren.

Die Auswahl der Kontrollgruppen ist wichtig in Hinblick auf die Frage der Kausalität. Es sollen dabei jene Unternehmen und Privatpersonen ausgewählt werden, die mehr oder weniger gleich wahrscheinlich am Programm teilnehmen würden, ohne dies auch wirklich zu tun.

Des Weiteren ist es für die Auswahl der Kontrollgruppe wichtig zu beachten, dass die Unternehmen und Privatpersonen, die an einem Programm teilnehmen, mit anderen nicht teilnehmenden Unternehmen und Privatpersonen verglichen werden, die ihnen in so vielen Parametern wie möglich ähneln, welche für die Signifikanz des Effekts des analysierten Programms wichtig sind.

Passende Methoden zum Erstellen einer Kontrollgruppe beruhen auf den beobachteten Charakteristika. Um eine entsprechende Kontrollgruppe für jedes Programm zu finden, sollte sich ein Teilnehmer so gut wie möglich an die Variablen oder bestimmenden Faktoren annähern, welche die Entscheidung des/der Einzelnen für die Teilnahme am Programm erklären.

Obwohl Unternehmen in beobachteten Charakteristika gut vergleichbar sind, können sie sich in unbeobachteten Charakteristika, z. B. Strategie, deutlich voneinander unterscheiden. Daher ist die Gruppe der teilnehmenden Unternehmen und Privatpersonen nicht unbedingt vergleichbar mit der Gruppe der nicht teilnehmenden Unternehmen und Privatpersonen. Resultierende Unterschiede zwischen den zwei Gruppen könnten den Unterschieden in den Firmencharakteristika, die mit dem Programm in Zusammenhang stehen, zugeschrieben werden. Die Korrelation zwischen Teilnahme und Resultat im Falle selektiven Gebrauchs ist daher eine unverlässliche Bewertung des kausalen Effekts des Programms.

Warum ist die Kontrollgruppe wichtig?

Das Resultat einer Effektmessung kann nicht besser sein als die Auswahl der Kontrollgruppe einer Evaluierung.

Folglich wird ein gewisser Standard basierend auf einem Minimum an beobachteten Charakteristika für die Entwicklung von Kontrollgruppen empfohlen. Auf diesem Weg wird eine Basis geschaffen, die es ermöglicht, ob es einen kausalen Zusammenhang gibt zwischen dem zu analysierenden Faktor und dem Performance-Ziel, gemeinsam mit der Basis für das Messen des isolierten Effekts, was allerdings auch vom zu analysierenden Programm abhängt. 


\begin{tabular}{|c|c|}
\hline $\begin{array}{l}\text { Minimale Voraussetzungen für die Auswahl einer } \\
\text { Kontrollgruppe für Unternehmen }\end{array}$ & $\begin{array}{l}\text { Minimale Voraussetzungen für die Auswahl einer } \\
\text { Kontrollgruppe für Privatpersonen }\end{array}$ \\
\hline $\begin{array}{ll}\text { - } & \text { Ausbildungsniveau der Angestellten } \\
\text { - } & \text { FuE Intensität } \\
\text { - } & \text { FuE Abteilung } \\
\text { - } & \text { Export Intensität } \\
\text { - } & \text { FuE Investitionen } \\
\text { - } & \text { Profit, Überschüsse oder Bruttogewinne } \\
\text { - } & \text { Industrie Zugehörigkeit }\end{array}$ & $\begin{array}{ll}\text { - } & \text { Ausbildung } \\
\text { - } & \text { Institution } \\
\text { - } & \text { Unternehmensgrösse } \\
\text { - } & \text { Industrie Zugehörigkeit } \\
\text { - } & \text { Anderechlecht und Alter } \\
& \text { Variable, wie z. B. Gehalt, Hintergrund, etc. }\end{array}$ \\
\hline
\end{tabular}

Es sollte erörtert werden, ob eine Kontrollgruppe ausschließlich aus FuE-aktiven Unternehmen bestehen soll oder ob innovative und nicht-innovative Unternehmen ebenfalls inkludiert werden sollen. Besteht die Kontrollgruppe ausschließlich aus FuE-aktiven Unternehmen, sollte dies gerechtfertigt werden, z. B. durch den Umstand, dass die analysierte Aktivität oder das analysierte Programm keine Aktivität ist, die Unternehmen über Nacht starten können, sondern, dass diese Aktivität ausschließlich auf FuE-aktive Unternehmen begrenzt ist.

Dies ist eine strenge Annahme, welche ohne Zweifel jene Unternehmen ausschließen würde, die a priori für die analysierte Aktivitäten festgelegt wurden. Im Gegensatz dazu könnte dies auch eine konservative Annahme sein, welche die Robustheit der Resultate garantiert, da sie einen Vergleich mit jenen Firmen vermeidet, in denen die Wahrscheinlichkeit der Teilnahme an den zur Frage stehenden Aktivitäten relativ gering ist. Durch das Sammeln von Input-Daten eines jeden regionalen und nationalen Forschungs- und Innovationsprogramms ist es möglich eine Kontrollgruppe aus Unternehmen oder Privatpersonen zu erstellen, die nicht an ähnlichen Programmen als das zu evaluierende Programm teilgenommen haben.

Die minimale Voraussetzung ist, dass so viele Charakteristika wie möglich in Betracht gezogen werden. Jedoch ist es wichtig zu viele erklärende Variable zu vermeiden, die entweder individuell oder in Kombination zu überlappenden Resultaten führen könnten. Werden zu viele identische Variable einbezogen, besteht das Risiko des Entstehens von Multikollinearität gemeinsam mit einer zu deutlichen Korrelation zwischen den erklärenden Variablen. Dadurch entstehen insignifikante Parameter, welche Resultate verfälschen. Beispielsweise, wenn FuE Intensität gemeinsam mit FuE Investitionen, FuE Abteilung und Unternehmensgröße eingeschlossen werden, da zwischen diesen Variablen Abhängigkeit besteht.

Es gibt unterschiedliche Wege eine Kontrollgruppe von Unternehmen und Privatpersonen auszuwählen, die mehr oder weniger die gleiche Wahrscheinlichkeit haben an einem Forschungs- und Innovationsprogramm teilzunehmen wie jene, die dies auch tun.

Oben haben wir eine zufällig ausgewählte Kontrollgruppe von Unternehmen und Privatpersonen mit untersuchten Charakteristika beschrieben. Die untersuchten Charakteristika können aber auch mit anderen Faktoren kombiniert werden.

Die Kontrollgruppe von Unternehmen und Privatpersonen kann aus jenen bestehen, die um Förderungen angesucht, diese jedoch nicht erhalten haben. Es können trotzdem Unterschiede zwischen den Top-Unternehmen oder Privatpersonen, die Förderung erhalten haben, und den schlechtesten Unternehmen oder Privatpersonen, die keine Förderung erhalten haben, bestehen, so dass die Kontrollgruppe trotzdem an Auswahl-Verfälschung und unbeobachteter Heterogenität leidet.

In den Fällen, wo die Bewerbungen eine Auswertung erhalten haben, könnte die Kontrollgruppe aus Unternehmen und Privatpersonen mit jenen Projekten bestehen, die unmittelbar innerhalb und 
außerhalb der Fördergrenze liegen, wo es sich um ähnliche ex ante Unternehmen und Privatpersonen handelt, ausgenommen in deren Wahrscheinlichkeit Förderung zu erhalten.

Eine andere Möglichkeit potentielle aber nicht teilnehmende Unternehmen und Privatpersonen für eine Kontrollgruppe zu finden, besteht durch Auslosung.

Dies stellt den einfachsten Weg dar, um den Effekt des Programms zu isolieren, da es dadurch total zufällig ist, wer eine Förderung erhalten hat und wer nicht. Diese Möglichkeit besteht für Forschungsund Innovationsprogramme mit einer Evaluierung plus einer Minimalauswertung aber ohne Rangfolge; oder ohne Evaluierung bzw. einer anderen Auswertung der Bewerbungen als zum Erfüllen der Administrationskriteria notwendig ist.

\subsubsection{Auswahl von Kontrollgruppen durch die so genannte "Propensity Score Nearest Neighbour Matching Methode"}

Basierend auf einer Vielzahl von Effektstudien und Forschunsartikeln, die von dänischen Forschern, FuE Konsulenten und dem dänischen Rat für Technologie und Innovation durchgeführt wurden, wird als Standard die "Propensity Score Nearest Neighbour Matching Methode" empfohlen. Diese wird dazu verwendet, die Gruppe der FuE-aktiven Unternehmen, die an einem Programm teilnehmen, und einer statistisch vergleichbaren Kontrollgruppe von FuE-aktive Unternehmen, die nicht teilnehmen, dies aber potentiell tun könnten, auf einer one-to-one Skala zu etablieren und abzugrenzen. Es ist unmöglich eine komplett identische Kontrollgruppe zu finden. ${ }^{9}$

Die Wahrscheinlichkeitsmodelle für die Teilnahme von Unternehmen an einem Instrument, die für die Identifizierung jener Faktoren verwendet werden, die darauf einen Effekt haben, ob die FuEaktiven Unternehmen im vorliegenden Instrument inkludiert sind, werden als logistische Regressionen gesetzt und in Verbindung mit der Propensity Score Matching Methode verwendet.

In den meisten Fällen wird es ein Vorteil sein eine Kontrollgruppe zusammen zu stellen, die so viele Kontroll-Unternehmen hat wie möglich - basierend auf dem Gesetz der großen Zahlen. Daher ist one-to-one eine Mindestvoraussetzung, der Standard sollte one-to-many sein. Des Weiteren sollten supplementierende Balance-Tests durchgeführt werden, um den Unterschied zwischen der Behandlungsgruppe und der Kontrollgruppe analysieren zu können.

Die so genannte Propensity Score Matching Methode wird in den Fällen angewendet, wo Unternehmen oder Privatpersonen, die an der analysierten Aktivität mit vergleichbaren FuE-aktiven oder innovativen Unternehmen oder Privatpersonen, die nicht an einer vergleichbaren Aktivität teilgenommen haben, abgestimmt werden. Die Idee dieser Methode ist, dass für ein Unternehmen T, die die gewünschte Aktivität durchführt, ein Unternehmen $C$ unter den anderen Unternehmen in der relevanten Statistik gefunden wird, die in einer Vielzahl von statistischen Parametern dem Unternehmen T durch das Aufzeigen der gleichen Wahrscheinlichkeit ('propensity score') an der relevanten Aktivität teilzunehmen ähneln, ohne dass $C$ reell an der Aktivität teilgenommen hat. Auf diese Weise kann Unternehmen T (als designiertes 'Behandlungs- oder Teilnahmeunternehmen') mit einem ähnlichen Unternehmen C (welches als 'Vergleichs- oder Kontrollunternehmen' designiert ist) verglichen werden, welches in der Statistik gefunden wurde. Statistisch soll Unternehmen C dem

Unternehmen T in den Faktoren Industrie, Unternehmensgröße, Export Muster,

\footnotetext{
${ }^{9}$ Examples of application of this method are found in DASTI (Innovation: Analyse og evaluering 01/2010):

"Productivity impact of business investments in research, development and innovation", DASTI (Innovation: Analyse og Evaluering 02/2011): "The economic impact of business-research collaboration", and DASTI (Innovation: Analyse og Evaluering 03/2013), "Economic impacts of Business Investments in R\&D in the Nordic Countries - A microeconomic analysis".
} 
Mitarbeiterentwicklung, Profit, Bruttogewinn und Zusammensetzung sowie FuE- oder Innovationsaktivitäten gleichen.

Was ist die empfohlene Methode eine Kontrollgruppe zu etablieren?

Die empfohlene Standardmethode ist die 'Propensity Score Nearest NeighbourMatching Method'. Andere Methoden wie die Verwendung von Reihungsinformation kann auch verwendet werden, allerdings liegt kein wissenschaftlicher Beweis vor, das seine Kontrollgruppe basierend auf Reihungsinformation eine bessere Kontrollgruppe begründet als die "Propensity Score Nearest NeighbourMatching Methode".

Andere Methoden wie die Etablierung von Kontrollgruppen basierend auf Information von der Reihung von Projekten im Evaluierungsprozess können ebenfalls verwendet werden. Der Vorteil dieser Methode ist, dass die Kontrollgruppe aus Beobachtungen von Unternehmen und Forschern besteht, die sich um die Teilnahme am Programm oder an der Initiative beworben haben, jedoch auf Grund diverser Evaluierungskriteria abgelehnt wurden. Jedoch sollten verständlicherweise nicht alle Bewerber in die Kontrollgruppe aufgenommen werden. Kontrollen sollten auf jenen Bewerbungen basieren, die beinahe die gleiche Auswertungszahl im Evaluierungsprozess erzielt haben, jedoch auf Grund finanzieller Limitierungen vom Förderungsprogramm abgelehnt wurden. Beobachtungen basierend auf der Behandlungs- und Kontrollgruppe sollten nur von jenen Projekten stammen, die sich knapp über und unter dem Grenzwert befinden, um die besten und schlechtesten Projekte von der Auswahl (sample) auszugrenzen.

Diese Herangehensweise bedarf eines exzellenten und zuverlässigen Reihungsystems, welches auf einer Vielzahl von untersuchten Projekten und von Beobachtungen nahe am Grenzwert basiert. Diese Art von Qualitätsdaten basierend auf Reihungsinformation sollte im EuroStars-Programm der Europäischen Union zu finden sein, in den Rahmenprogrammen der Europäischen Union und in den Innovationskonsortia-Programmen der Dänischen Agentur für Wissenschaft, Technologie und Innovation. Es liegt jedoch kein wissenschaftlicher Beweis vor, dass eine Kontrollgruppe basierend auf High-quality-ranking-Beobachtungen eine bessere Kontrollgruppe begründet als die "Propensity Score Nearest Neighbour Matching Methode".

Als natürliche Folge ist es nicht möglich beim Gebrauch dieser oder anderer Methoden eine Kontrollgruppe zu finden, die in allen teilweise nicht-beobachtbaren Faktoren vollständig identisch ist. Durch Verwendung einer anderen ausgewählten Kontrollgruppe können durchaus unterschiedliche Resultate erzielt werden. Deswegen ist es wichtig, die Charakteristika, die in der Kontrollgruppe gefunden werden, interpretieren zu können.

3.3 Grundsatz 3 - Verwendung der difference-in-difference Methode und balancierte Paneldaten

Die Möglichkeiten hängen vom Design der innovationspolitischen Instrumente ab.

Zum Beispiel könnten manche innovationspolitischen Instrumente sich für bedeutend präzisere Schätzwerte der Effekte öffnen als bei der Matching Methode (beschrieben weiter oben) und der difference-in-difference Methode (beschrieben weiter unten). Das hängt zum Beispiel davon ab, ob ein Regressions-Diskontinuitätsdesign möglich ist. 


\subsubsection{Die difference-in-difference Methode}

Eine der empfohlenen gängigen zentralen statistischen Methoden ist die difference-in-difference Methode. Diese Methode kommt dann zur Anwendung, wenn Unterschiede in der Entwicklung in der Behandlungsgruppe und Kontrollgruppe von statistisch identischen Unternehmen und Privatpersonen ohne analysierter Aktivität errechnet werden sollen. ${ }^{10}$

Die difference-in-difference Methode basiert auf dem Vergleich von Wechsel in Resultaten (Performance-Ziel). Das Model sieht wie folgt aus:

steht für den Effekt der Aktivität, welcher auf Basis des Unterschieds zwischen der Entwicklung in den Performance Indikatoren errechnet wird, genannt Y, gemessen an der Behandlungsgruppe (T), definiert als Performance Indikator zum Zeitpunkt 1 minus dem Performance Indikator zum Zeitpunkt 0, und der Entwicklung in den Performance Indikatoren der Kontrollgruppe (C), definiert als der Performance Indikator zum Zeitpunkt 1 minus Performance Indikator zum Zeitpunkt $0 . \mathrm{Ob}$ ein signifikanter Unterschied vorliegt zwischen diesen beiden kann anhand Standard T-Tests oder linearer Regression getestet werden.

\section{Box 1 Zentrale Analyse Methode: Difference-in-difference}

Difference-in-difference:

(a) Davor-danach Vergleich von Firmen die am Programm teilnehmen (Teilnehmer)

(b) Davor-danach Vergleich von Unternehmen die nicht am Programm teilnehmen (Kontrollgruppe)

Evaluierung ob (a) positive ist als (b).

T1 - Erfolgsparameter des Teilnehmers davor.

T2 - Erfolgsparameter des Teilnehmers danach.

C1 - Erfolgsparameter des nicht-Teilnehmers davor.

C2 - Erfolgsparameter des nicht-Teilnehmers danach.

Der Differenz (T2-T1)-(C2-C1) errechnet die Differenz der Zunahmen.

\section{Was ist die Schlüsselmethode für Effekt-Analysen?}

The Schlüsselmethode für Analysen des Unterschieds zwischen den Teilnehmern an der politischen Initiative und der Kontrollgruppe, die nicht teilnimmt, ist die difference-in-difference Methode.

\footnotetext{
${ }^{10}$ Beispiele für die Anwendung diese Models können im DASTI (Innovation: Analyse og evaluering 01/2010) gefunden werden: "Productivity impact of business investments in research, development and innovation", and DASTI (Innovation: Analyse og Evaluering 02/2011): "The economic impact of business-research collaboration".
} 


\subsubsection{Balancierte Paneldaten}

Der Effekt von Unternehmensinvestitionen in FuE auf Mehrwert und Produktivität per Angestellten ist ein dynamischer Prozess, der mit der Zeit variieren kann. Sektionsübergreifende Analysen, die auf einem einzelnen Jahr basieren, sind nicht adäquat für eine Analyse der Variation über Zeit.

Des Weiteren können nicht beobachtbare Effekte bei einem individuellen Unternehmen auftreten, die die Modelle nicht erfassen können. Der Davor-danach-Vergleich, der von der Anwendung der difference-in-difference Methode resultiert, bedeutet, dass Paneldaten (Sektoren übergreifende Daten über Zeit) und Methoden notwendig sind, um diese nicht beobachtbaren Effekte messen zu können.

Grosse Unternehmen werden jedes Jahr in die Forschungs- und Entwicklungsstatistiken einbezogen, während Proben von KMUs zufällig ausgewählt werden. Das Resultat ergibt ein sehr ,schlecht balanciertes ' Panel. Für einige Unternehmen liegen Beobachtungen aller Jahre vor, während bei andern Daten fehlen. Deshalb wird die Zusammensetzung der Paneldaten wie folgt empfohlen:

- Paneldatenanalysen sollten nur an Unternehmen mit mindestens zwei Beobachtungen durchgeführt werden. Um abzusichern, dass die Analysen so repräsentativ wie möglich sind, sollten alle Unternehmen mit zwei oder mehreren Beobachtungen mit einbezogen werden. Wenn es die Datenbank zulässt, sollten die Anforderungen gehoben werden, so dass nur Unternehmen mit drei oder mehreren Beobachtungen mit einbezogen werden. Dies wird die Anzahl der Unternehmen in den Analysen natürlich reduzieren.

- Folgende Herangehensweise wird für fehlende Beobachtungen in Zeitserien empfohlen: Fehlt nur ein Beobachtungsjahr in einer Zeitserie, sollte dieses geschätzt werden. Fehlen zwei oder mehrere, sollte der letzte zusammenhängende Teil der Zeitserie behalten werden.

- Extensive Änderungen in den Variablen könnten eine Unternehmensfusion oder Unternehmensteilung indizieren. Solche Änderungen sollten einen unverhältnismäßig größeren Effekt auf die Resultate haben. Es wird empfohlen den internationalen Standards zu folgen und jene Unternehmen, deren jährliche Wachstumsrate in Mehrwert, Anlagevermögen, Zahl der Angestellten oder FuE Kapital von weniger als -50\% oder mehr als 300\%, zu entfernen.

- Es wird empfohlen Sensitivitätsanalysen durchzuführen wenn Grunddaten geändert werden.

\subsubsection{Standards für die Berechnung von ökonomischen Effekten}

Die Cobb Douglas Produktivitätsfunktion gilt als Standard für das Anzeigen des Effekts eines gegebenen Instruments in Pfund und Pence in der Form von gesteigerter Produktivität per Angestellten, Profit, etc. Dies wird typischerweise als OLS Regression modelliert. ${ }^{11}$

Abhängig vom ausgewählten KPI (der analysierten Erfolgsvariable), der Änderungen des Levels über Zeit, könnten auch die jährliche Änderung der Wachstumsrate über Zeit sein. Als Beispiel von Änderungen von Levels könnte die Änderung der Zahl der Angestellten und der Level der Anstellung gelten.

Ein Beispiel relativer Änderung gilt die Überlebensrate von Unternehmen oder die Anstellungsquote. Beispiele von Änderungen der Wachstumsrate sind das Wachstum an Produktivität per Angestellten, die Umsatzsteigerung oder das Wachstum des Unternehmensmehrwerts. Generell hängt der Standard für die Berechnung des ökonomischen Effekts von den Key Performance Zielen ab, die beurteilt und geschätzt werden.

\footnotetext{
${ }^{11}$ Beispiele für die Anwendung diese Models können im DASTI (Innovation: Analyse og Evaluering 02/2011) gefunden werden: "The economic impact of business-research collaboration", and DASTI (Innovation: Analyse og Evaluering 03/2013), "Economic impacts of Business Investments in R\&D in the Nordic Countries - A microeconomic analysis".
} 
Bei der Auswahl von Hintergrundfaktoren ist es wichtig zu beachten wie der individuelle Hintergrundfaktor sowohl Resultat als auch Behandlung beeinflusst. Zum Beispiel könnte eine Zeitrelatierte Herausforderung auftreten, die von der Behandlung eines Modells mit verzögerten Variablen betroffen sein kann.

\subsection{Grundsatz 4 - Behandlung von Außenseitern}

Außenseiter sind Beobachtungen, die vom Muster aller anderen Beobachtungen deutlich abweichen. Um so repräsentative Resultate wie möglich erzielen zu können, sollten ökonometrische Modelle in der Lage sein, Effekte in einer breiten Auswahl an Unternehmen zu messen. Jedoch können extreme Werte die Effekte stören und dadurch die Präzision vermindern. In manchen Fällen gibt es gute Gründe um diese extremen Werte zu entfernen. Als Beispiel kommt es bei jungen Unternehmen oft vor, dass große und riskante Investitionen vorgenommen werden, die den Unternehmens-Mehrwert für eine kurze Zeit stark beeinflussen. Solche Firmen können potentiell ein extremes Wachstum von einem zum anderen Jahr aufweisen.

$\mathrm{Ob}$ Extremwerte entfernt werden sollen, hängt vom Zweck der Analyse und dem innovationspolitischen Instrument ab. Deshalb sollte in jeder Analyse und jedem Instrument sorgfältig überlegt werden, ob Außenseiter entfernt werden sollen.

Des Weiteren wurden Daten registriert, die Extremwerte von beispielsweise durchschnittlicher Unternehmsproduktivität per Angestellten, Mehrwert, Anstellung, etc darstellen. Dabei handelt es sich um inkorrekte Registrierungen, die entweder mit dem Unternehmensmehrwert oder der Zahl der volljahres Äquivalenten zusammenhängen. Unabhängig davon wo die inkorrekte Registrierung gefunden wird, wird empfohlen, solche Werte zu entfernen. Es gibt jedoch andere Methoden, um beispielsweise Extremwerte ein- oder auszuschließen, um zu sehen, ob dies auf das Resultat Auswirkungen hat oder um Mittelwerte zu überlegen.

\subsection{Grundsatz 5 - Langzeit Serien}

Effektmessung ist complex, da eine lineare Verbindung zwischen der analysierten Aktivität und einem folgenden Effekt kaum gefunden werden kann. Deshalb gibt es seine Vielzahl an Bedingungen, die eine Effektmessung erschweren könnten, wie z. B. potentielle Zeitabschnitte bevor der Effekt eintritt, unterschiedliche Startzeiten für die Unternehmen, Unterschiede zwischen Unternehmenscharakteristika und -Erfahrung in Hinsicht auf das Instrument.

Als Standard müssen die ökonometrischen Modelle in der Lage sein Zeitverzögerungen zwischen der analysierten Aktivität und dem daraus resultierenden Effekt zu erlauben. Der Effekt kann mit variierender Verspätung einsetzen.

\subsection{Grundsatz 6 - Robustheits Test}

Die Forscher untersuchten die Robustheit der Resultate in Hinblick auf verschiedene übereinstimmende Prozesse. Sie unterstreichen dabei, dass ihre Schätzungen durch unbeobachtete Charakteristika verfälscht sein könnten. In der Tat können beim Gebrauch von übereinstimmenden Methoden niemals Verfälschungen - verursacht durch unbeobachtete Variablen -ausgeschlossen werden, was deren Haupteinschränkung ist.

\subsection{Grundsatz 7 - Effektindikatoren sollten relativiert werden}

Es ist nicht immer einfach Effekte zu identifizieren und einzugrenzen. Des Weiteren entstehen Unterschiede in der Bewertung abhängig von Akteuren und Interessenvertretern, z. B. bezogen auf den Marktwert eines Unternehmens. Ein möglicher Vorschlag wäre eine Marktbewertung des individuellen Unternehmens zu verwenden, als Maßnahme für den Preis oder Wert der gesamten 
'materiellen' und 'immateriellen' Vermögenswerte. Dies setzt jedoch voraus, dass die analysierten Unternehmen an der Börse notiert sind. Da aber nur sehr wenige Unternehmen an der Börse notiert sind, kommt diese Methode nicht zur Anwendung.

Wenn Effekte in Unternehmen analysiert werden, wird empfohlen einen KPI zu verwenden, welcher in Relation zum Arbeitskraft-Input relativiert wurde. Dadurch wird sichergestellt, dass die Effekte nicht dem endlosen Angebot an Arbeitskraft angerechnet werden können.

\subsection{Grundsatz 8 - Peer Review der Resultate}

Die Qualität der Effektmessungen müssen mit unabhängigen Forschungsorganisationen, die nicht an der Analyse teilgenommen haben, diskutiert werden, z. B. durch die Etablierung von Lenkungsausschüssen oder anderen Peer Reviews, Forschungsseminaren, Workshops mit politischen Entscheidungsträgern, etc. Ziel ist es Effektmessungen auf Grundlage der besten und am weitesten anerkannten internationalen Forschungs- und Statistikmethoden durchzuführen. Vorzugsweise sollten die Resultate der Effektmessungen für die Publizierung in angesehenen internationalen Fachzeitschriften oder an großen internationalen Konferenzen geeignet sein.

\subsection{Grundsatz 9 - Fehler und Stress Tests}

Effektstudien tragen auch zum politischen Lernen bei. Politische Entscheidungsträger und Programmmanager sollten kritische Kernpunkte, 'Stress Tests' des Programms, fehlerhafte Resultate, Scheitern von Projekten und Programmen, etc. als Teil der Messung akzeptieren. Der Auftraggeber/die Auftraggeberin der Messung sollte von Fehlern lernen und nicht nur auf Erfolgsgeschichten aus sein. Eine enge Beziehung und ein hohes Level an Interaktion und gemeinsamen Vertrauens zwischen Programmsponsoren und Gutachtern steigern die Wahrscheinlichkeit der Formulierung und Umsetzung einer effektiven und brauchbaren Messung, brauchbar in dem Sinn, dass die Studie zum politischen Lernen und neuem Wissen über die Stärken und Schwächen des Programmes beigetragen hat. Die Effektanalysen sollten nicht nur auf jene Gebiete und Effektindikatoren begrenzt sein, wo ein Erfolg des Programms sehr wahrscheinlich ist; Unternehmer und Programmmanager sollten auch nach Fehlern und in den Gebieten suchen, in denen das Programm nicht die erwünschten Effekte und Aktivitäten erzielt.

Obwohl CIM 2.0 Grundsätze und ein Minimum an Standards für Effektmessungen enthält, wurde er mit Bedacht auf Raum für Flexibilität verfasst. Der Grund dafür ist, dass die empfohlene "Propensity Score Nearest Neighbour Matching Methode" nicht für alle Arten von Forschungs- und Innovationsprogrammen und politischen Initiativen die beste Methode darstellt. Dies wird dann der Fall sein, wenn zum Beispiel die direkte Business-bezogenen Performance Ziele nicht im Mittelpunkt der Analyse stehen, sondern dort, wo die Studie einen breiteren Fokus hat. Im Falle anderer Programme und Initiativen wird es weniger relevant sein Effekt-Evaluierungen der aufgelisteten ökonomischen Performance Ziele durchzuführen, da die Hauptziele eines Programms und Initiative andere nicht-ökonomische Aktivitäten sein könnten.

Dies ist der Fall, wenn beispielsweise Effektmessungen in Verbindung mit Cluster und Innovationsnetzwerken, Forschungsplattformen und Netzwerke, etc., wo die Hauptziele nicht notwendigerweise ökonomische Performance Ziele alleine, sondern auch nicht-ökonomische verhaltensregulierende Performance Ziele sind. Daher ist es wichtig, dass konkrete Effektmessungen berücksichtigen, was das Ziel eines gegebenen Programms oder Initiative ist.

Gleichzeitig haben die meisten Länder nicht die gleichen Möglichkeiten wie Dänemark, Norwegen, Schweden und die Niederlande, auf Grund limitiertem Zugangs zu national deckenden quantitativen Micro-Daten und langen Zeit Serien. Für die Mehrheit der Länder ist es schwierig die Micro-Daten 
für die gesamte Wirtschaftsbasis zu entwickeln, die für solide und zuverlässige quantitative ökonometrische Analysen die Effekte von wirtschaftsbezogene Forschungs- und Innovationspolitik historisch dokumentieren und berechnen können.

Aus diesem Grund beinhaltet das Handbuch einen Überblick über die nicht-ökonomischen Performance-Indikatoren der wichtigsten Forschungs- und Innovationsprogramme und politischen Initiativen des Ministeriums für Forschung und weiterführende Bildung.

Das dänische Ministerium für Forschung und weiterführende Bildung führte den Vorsitz einer internationalen Arbeitsgruppe über Performance-Indikatoren im Zuge des EU Pro-INNO Projekts EPISIS. Diese Arbeitsgruppe hatte Vertreter aus insgesamt 8 Regierungen aus den Ländern Dänemark, Schweden, Norwegen, Slowenien, Österreich, Deutschland, Großbritannien und Finnland als auch von der Europäischen Kommission. Die besten Beispiele für Messungen und PerformanceIndikatoren wurden ausgetauscht und ein Handbuch über Empfehlungen für Indikatoren besprochen, welches für das Aufstellen von Performance-Ziele und KPIs verwendet werden kann. ${ }^{12}$

Der EPISIS Report von 2011 über Effektanalysen und Messungs-Indikatoren war ebenfalls ein integrierter Teil der Arbeit einer Gruppe von politischen Entscheidungsträgern, Programmmanagern und Forschern aus Dänemark, Estland, Finnland, Deutschland, Island, Litauen, Norwegen, Polen, Schweden, Großbritannien und den USA, die sich 2011 und 2012 um ein gemeinsames Verständnis für Cluster und Netzwerkpolitik und ein weltweit führendes Benchmarking, sowie Überwachungsund Effektmessungs-Tools und Indikatoren zu entwickeln. Als Resultat dieser Arbeit wurden eine Reihe von Empfehlungen für mögliche KPIs, Effektmessungmethoden, Benchmarking and Überwachungstools in der Publikation "Let's make a perfect cluster policy and cluster programmesmart recommendation for policy makers" verfasst. Basierend auf internationale best practice wurde ein ausgezeichnetes Messungsbündnis bestehend aus Benchmarking, Überwachung und Effektanalysen in den drei folgenden Levels in der Publikation empfohlen. ${ }^{13}$

\subsection{Ex ante Messung}

Eine Darstellung von Zielen und erwarteten Effekten des Programms sollte für jedes Forschungs- und Innovationsprogramm in separaten Performance-Beschreibungen gegeben sein. Demnach soll die Performance-Beschreibung unter anderem eine ex ante Messung des Programms beinhalten.

Auf dieser Grundlage schreibt die Dänische Agentur für Wissenschaft, Technologie und Innovation KPIs für jedes Forschungs-Innovationsprogramm vor. Diese Indikatoren können KPIs in Form von sogenannten Investitions- (Input), Aktivitäten- (Output), Resultats- (Outcome) und Effekt- (Impact) Indikatoren sein. Es ist wichtig die KPIs bereits im Design jedes neuen Forschungs- und Innovationsprogramms vorzuschreiben und nicht erst dann, wenn ein Programm gemessen werden muss.

Die Messungen und Empfehlungen für die Auswahl der Indikatoren folgen dem Werk des EPISIS Projekts ${ }^{14}$ und dem Werk in Verbindung mit den Empfehlungen für Clusterprogramme und Politik ${ }^{15}$, welche auf die Innovation im Servicebereich und dem Erstellen von effektiver FuE und

\footnotetext{
${ }^{12}$ EPISIS Report (2011), Service innovation: Impact Analysis and Assessment Indicators.

${ }^{13}$ Gerd Meier zu Köcker, Thomas Lämmer-Gamp und Thomas Alslev Christensen: "Let's make a perfect cluster policy and cluster programme - smart recommendation for policy makers," Berlin/Copenhagen 2012, VDI/VDE-IT and DASTI, October 2012, page 39-47.

${ }^{14}$ EPISIS Report (2011), Service innovation: Impact Analysis and Assessment Indicators.

${ }^{15}$ Gerd Meier zu Köcker, Thomas Lämmer-Gamp and Thomas Alslev Christensen: "Let's make a perfect cluster policy and cluster programme - smart recommendation for policy makers," Berlin/Copenhagen 2012, VDI/VDE-IT and DASTI, October 2012, page 39-47.
} 
Innovationsclusterprogrammen abzielen. Es kann aber auch als Inspiration sowohl anderen Programmen als auch nationaler Gesetzgebung dienen.

\subsubsection{Input und Output-Indikatoren}

Das Ziel der Input (Investitions-) Indikatoren in Bezug auf FuE und Innovation ist es, den nützlichen Akt zu erfassen, den ein Unternehmen tätigt um Innovation zu erzeugen. Demnach messen InputIndikatoren den vorsätzlichen Teil des Innovationsprozesses in Unternehmen und stellen Resourcen und Bemühen, welche Unternehmen für den Prozess aufbringen, dar.

Es gibt viele Quellen des vorsätzlichen Teils eines Innovationsprozesses, und die Input- und OutputIndikatoren sind in Gruppen entsprechend aufgeteilt, die Management, Angestellte, Netzwerke, etc. vertreten. Es ist ein wichtiger Punkt, dass die Input Indikatoren messen sollten, ob Unternehmen vorsätzlich Innovation vorantreiben, und dass dieser Prozess nicht notwendigerweise zu Innovation führt. Deswegen sollte ein Indikator nicht danach beurteilt werden, ob das Resultat auch wirklich Innovation mit sich bringt, sondern ob Input Indikatoren die Vorsätzlichkeit zur Innovation im Prozess eines Unternehmens messen.

\subsection{Basismaßnahmen für ex post Messungen}

Der Fokus liegt auf der Sicherung von Basismaßnahmen der Bemühungen, um die Situation vor dem Launch von Forschungs- und Innovationsprogrammen (und jender Situation, in der die Programme gar nicht existieren) zu dokumentieren, damit der Effekt des Forschungs- und Innovationsprogramms in Verbindung mit der Situation geschätzt werden, kann falls das Programm nicht umgesetzt wird.

In dieser Verbindung werden die neuesten forschungsbasierten Methoden durch das Wählen von Kontrollgruppen im Voraus angewandt, die die Situation so widerspiegeln, als wäre das Programm nicht umgesetzt worden. Falls die Analyse eine genügend große Zahl an Indikatoren aufweist, kann die propensity score matching Methode angewendet werden, um Basismaßnahmen erstellen zu können (siehe Grundsatz 2). Auf dieser Basis können ex post Messungen mit Schätzungen des Effekts des Programms ausgeführt werden.

Auf dieser Grundlage legt die Dänische Agentur für Wissenschaft, Technologie und Innovation KPIs für jedes Forschungs- und Innovationsprogramm. Diese können KPIs in Form von sogenannten Outcome- (Resultat) und Impact (Effekt) Indikatoren sein. 
Tabelle 4.1 Input, output, outcome and impact Indikatoren in den Effektmessungen.

\begin{tabular}{|c|c|c|c|}
\hline & & Outcome & Impact \\
\hline $\begin{array}{l}\text { Investitione } \\
\text { Entwicklur }\end{array}$ & & & \\
\hline  & 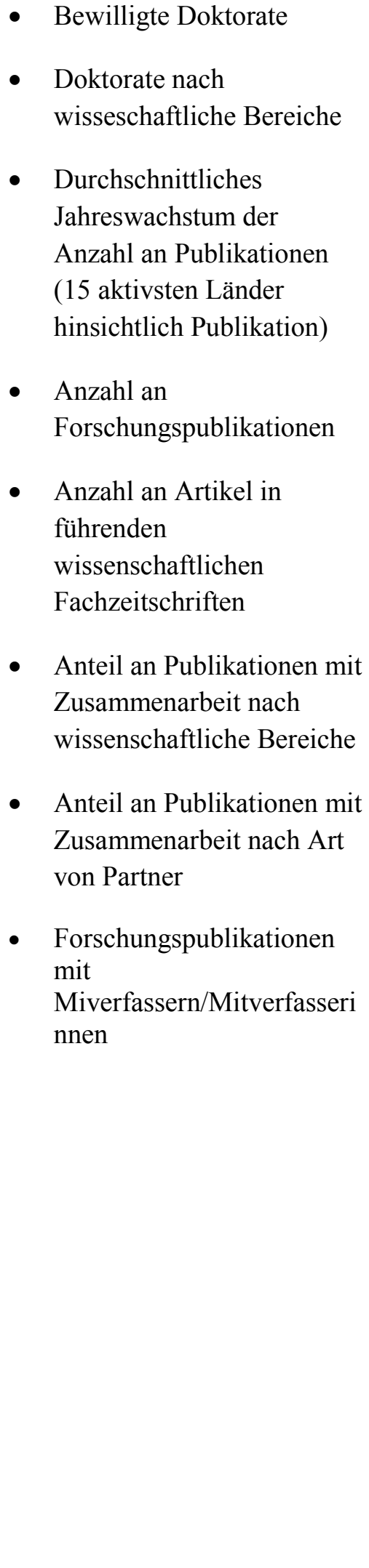 & $\begin{array}{l}\text { - Zitate von } \\
\text { wissenschaftlichen } \\
\text { Fachzeitschriften } \\
\text { - } \\
\text { Effekt von } \\
\text { Publikationen in } \\
\text { Hinblick auf } \\
\text { wissenschaftliche } \\
\text { Bereiche (Nordische } \\
\text { Länder) } \\
\text { EPO-Patente } \\
\text { - Lizenzvereinbarungen } \\
\text { öffentlicher } \\
\text { Forschungsinstitute, } \\
\text { Patentansuchen und } \\
\text { Spin-Outs } \\
\text { - } \\
\text { Anteil an } \\
\text { Unternehmen mit } \\
\text { Innovationsaktivitäte } \\
\text { neue Technologien } \\
\text { - } \\
\text { Anteil an PP } \\
\text { innovativen } \\
\text { Unternehmen } \\
\text { Anteil an PP } \\
\text { innovativen } \\
\text { Unternehmen mit } \\
\text { innovativer } \\
\text { Kooperation } \\
\text { Die 100 FuE } \\
\text { aktivsten } \\
\text { Unternehmen („EU } \\
\text { Industrial R\&D } \\
\text { Scoreboard") } \\
\text { - }\end{array}$ & $\begin{array}{l}\text { - Wachstum } \\
\text { von } \\
\text { Reallöhnen } \\
\text { - BNP } \\
\text { Wachstum } \\
\text { - Wachstum der } \\
\text { Beschäftigung } \\
\text { - Wachstum } \\
\text { des } \\
\text { Handelsumsat } \\
\text { zes oder von } \\
\text { Bruttogewinn } \\
\text { en } \\
\text { Lösungen der } \\
\text { Probleme der } \\
\text { Gesellschaft } \\
\text { (Gesundheit, } \\
\text { Umwelt, } \\
\text { Energie, } \\
\text { Klima, } \\
\text { Wohnen, } \\
\text { Armut, } \\
\text { Bildung....) }\end{array}$ \\
\hline
\end{tabular}




\subsubsection{Outcome und Impact Indikatoren ${ }^{16}$}

Outcome (Resultat)-Indikatoren beschäftigen sich mit dem Neuwert der Innovation. Sie messen das intermediäre Resultat des Innovationsprozesses. Aus diesem Grund handelt der Outcome mehr von Wertschaffungsaktivitäten als von der eigentlichen Werterfassung des Innovationsprozesses, welche mit Impact Indikatoren gemessen werden. Die Impact (Effekt)-Indikatoren versuchen den Wert zu messen, den eine Service-Innovation für das Unternehmen, Kunden und die Gesellschaft erzeugt. Für das Unternehmen kann sich der Wert der Serviceinnovation darin auswirken, dass einerseits den Kunden ein neues Service angeboten werden kann, andererseits Kosten und betriebsinterne Ausgaben gesenkt werden können. Dem Kunden kommt der Wert vom Angebot eines verbesserten Services im Vergleich zu den existierenden Angeboten oder von einem neuen Service auf dem Markt zugute, weil er somit aus einer größeren Vielfalt an Services auswählen kann.

Letzteres ist in der akademischen Literatur über internationalen Handel unter dem Konzept "love of variety" bekannt (Krugman, 1979b). Der gesteigerte Wert für die Gesellschaft folgt dem Wert für Unternehmen und Kunden. Ist ein Unternehmen demnach in der Lage z. B. seine Produktivität zu steigern, ist dies wiederum wertvoll für die Gesellschaft durch ein wachsendes BNP. Des Weiteren erhöht Innvoation von Unternehmen den Marktwettbewerb und stellt sicher, dass das stärkste Unternehmen überlebt.

\subsection{Resultate von Effektmessungen in Dänemark}

Seit 2008 wurden mehr als 17 Impact-Messungen von verschiedenen Forschungs-, Innovations- und Unterrichtsprogrammen durchgeführt. Die Effektmessungen wurden von unabhängigen Forschern oder Organisationen durchgeführt und wurden vom Ministerium, den Behörden, dem Forschungsrat oder unabhängigen Institutionen in Auftrag gegeben. 12 Haupt-Effektmessungen von innovationspolitischen Instrumenten wurden alleine 2010 und 2013 durchgeführt ${ }^{17}$. Des Weiteren wird erwartet, dass DASTI 5 neue Impact Studien im Jahr 2014 publiziert.

Folgende sind Beispiele von Effektmessungen: Impact auf die Produktivität dänischer BusinessR\&D und Innovationsinvestments, Programm der Innovationskonsortia, Wissenspilot (Innovation Assistant scheme), Inkubatorprogramm, Industrie-Doktorat Programm, Innovation Networks

Denmark („Clusterpolitik“) Programm, EUREKA Projekte und Business-Forschungs Kollaborationsprojekte zwischen Universitäten und Unternehmen) und dem dänischen GTS-System (Forschungs- und Technologieeinrichtungen).

\footnotetext{
${ }^{16}$ EPISIS Report (2011), Service innovation: Impact Analysis and Assessment Indicators.

${ }^{17}$ http://fivu.dk/en/publications/2012/central-innovation-manual-on-excellent-econometric-impact-analyses-ofinnovation-policy-cim
} 
Box 5.2. Übersicht über Effektmessungen von Programmen und Politischen Initiativen

\begin{tabular}{|c|c|}
\hline Fokus & Cluster- und Netzwerkpolitik \\
\hline $\begin{array}{l}\text { Studie Nr. } 1 \\
\& \text { Studie Nr. } \\
18\end{array}$ & $\begin{array}{l}\text { Die unabhängige Impact Messung von Cluster-Politik in Dänemark (The Innovation Networks } \\
\text { Denmark Programme) (DASTI 18/2011): Die Programme unterstützen die Etablierung und den } \\
\text { Betrieb von Cluster- und Netzwerkorganisationen. Aus } 1200 \text { nicht-innovativen am Prgramm } \\
\text { teilnehmenden Unternehmen stieg die Wahrscheinlichkeit innovativ zu werden um } 300 \text { Prozent im } \\
\text { Vergleich zu } 1200 \text { statistisch identischen Unternehmen, die nicht von der Infrastruktur des } \\
\text { Innovationsnetzwerks Dänemark Gebrauch gemacht haben. Unter den R\&D-aktiven oder } \\
\text { innovativen Unternehmen, die am Programm teilgenommen haben, stieg die Wahrscheinlichkeit } \\
\text { ihr erstes R\&D Kollaborationsprojekt mit einem Forschungsinstitut in die Wege zu leiten um } 300 \\
\text { Prozent im Vergleich zu statistisch identischen Unternehmen, die nicht am Programm } \\
\text { teilgenommen haben. } \\
\text { Der Impact auf das Wachstum der Produktivität des dänischen Innovations- und Research } \\
\text { Support Systems (DASTI 02/2014, April 2014): Eine unabhängige Meta-Messung (siehe Studie } \\
\text { Nr. 18) entdeckte, dass Unternehmen, die am Innovationsnetzwerksprogramm teilgenommen } \\
\text { haben, in den folgenden zwei Jahre hinsichtlich Gesamt-Faktorproduktivität im Durchschnitt um } 4 \\
\text { Prozentpunkte schneller wachsen als ähnliche nicht-teilnehmende Unternehmen. }\end{array}$ \\
\hline Fokus & R\&D Kollaborationsprojekte zwischen Wirtschaft und Forschung \\
\hline $\begin{array}{l}\text { Studie Nr. 2- } \\
4 \text { und Studie } \\
\text { Nr. } 18\end{array}$ & $\begin{array}{l}\text { Drei unabhängige Effekt Messungen (DASTI 06/2008, DASTI 03/2010, DASTI 01/2011 und } \\
\text { Kaiser \& Kuhn (2012)) des dänischen Innovations-Konsortiumsprogramm (öffentliche } \\
\text { Fördermittel für große Forschungs-Kollaborationsprojekte zwischen einigen Unternehmen und } \\
\text { Forschungsinstitutionen und Technologie-Instituten) zeigen statistisch signifikante Effekte sowohl } \\
\text { für Unternehmen als auch für individuelle Forscher abhängig von den zu untersuchenden Key } \\
\text { Impact Indikatoren. Key Performance Indikatoren sind Brutto-Gewinn, individuelle Beschäftigung, } \\
\text { Beschäftigung generell, und Beschäftigung in Unternehmen, Patentaktivität, Gehälter und Gesamt- } \\
\text { Faktorproduktivität. Teile der Analyse zeigen einen positiven und satistisch signifikanten Impact } \\
\text { für kleine und mittlere Unternehmen hinsichtlich Arbeitsproduktivität, Patentaktivität und } \\
\text { Beschäftigung. Keine zeigt Impact auf Gesamt-Faktorproduktivität oder auf große Unternehmen. } \\
\text { Eine Studie zeigt einen positiven, statistisch signifikanten Impact auf das Gehaltsniveau der } \\
\text { Forscher an den Forschungsinstituten. Der Brutto-Gewinn stieg durchschnittlich auf } 2.7 \text { Millionen } \\
\text { EUR bei den Teilnehmern am Innovations-Konsortium über einen Zeitraum von neun Jahren nach } \\
\text { dem Beginn des Innovations-Konsortiums. Unternehmen haben keine öffentlichen Fördermittel } \\
\text { erhalten. } \\
\text { Der Impact auf das Wachstum der Produktivität des dänischen Innovations- und Research } \\
\text { Support Systems (DASTI 02/2014, April 2014): Eine unabhängige Meta-Messung (siehe Studie } \\
\text { Nr. 18) entdeckte, dass Unternehmen, die am Innovations-Konsortiumsprogramm teilgenommen } \\
\text { haben, in den folgenden zwei Jahre hinsichtlich Gesamt-Faktorproduktivität im Durchschnitt um } \\
\text { 3,5 Prozentpunkte schneller wachsen als ähnliche nicht-teilnehmende Unternehmen. }\end{array}$ \\
\hline Studie Nr. 5 & $\begin{array}{l}\text { Eine unabhängige Impact Messung (DASTI 17/2011) von international Forschungs- und } \\
\text { Entwicklungs-Kollaborationsprojekten (EUREKA-projects) wurde } 2011 \text { durchgeführt. Darin } \\
\text { wurde der Impact der EUREKA-Teilnahme hinsichtlich Arbeitsproduktivität, Beschäftigung, } \\
\text { Umsatz und Exporte analysiert. Das Ergebnis zeigt einen positiven, statistisch signifikanten Impact } \\
\text { auf Wachstumsraten der Arbeitsproduktivität, Beschäftigung, Umsatz und Exporte im Vergleich zu } \\
\text { statistisch ähnlichen Unternehmen, die nicht an EUREKA-Projekten teilgenommen haben. } \\
\text { EUREKA-Teilnahme resultiert auch in significant höheren Wachstumsraten der Exporte und } \\
\text { Beschäftigung verglichen mit Unternehmen, die ausschliesslich am Innovations- } \\
\text { Konsortiumsprogramm teilgenommen haben (und nicht an internationalen Projekten). }\end{array}$ \\
\hline
\end{tabular}




\begin{tabular}{|c|c|}
\hline Studie Nr. 6 & $\begin{array}{l}\text { Eine unabhängige Effekt Messung (DASTI 02/2011) von nationalen Forschungs- und } \\
\text { Innovations-Kollaborationsprojekten zwischen Unternehmen und Universitäten oder GTS- } \\
\text { Instituten (auf Dänisch) wurden } 2010 \text { und } 2011 \text { durchgeführt. Unter Projekte werden jene mit und } \\
\text { ohne Unterstützung öffentlicher Fördermittel verstanden. Mehr als } 1500 \text { FuE-aktive Unternehmen, } \\
\text { die sich an einem oder mehreren FuE-Kollaborationsprojekten mit Forschungs- und Technologie- } \\
\text { Institutionen in der Zeit 1999-2006 beteiligt haben, wurden mit mehr als } 1500 \text { statistisch } \\
\text { identischen Unternehmen ohne Kollaboration verglichen, die unter } 20000 \text { dänischen FuE-aktiven } \\
\text { Unternehmen ermittelt wurden. Die Arbeitsproduktivität ist bei durchschnittlichen Unternehmen } \\
\text { mit FuE-Kollaboration um } 9 \text { Prozent höher als bei statistisch identischen FuE-aktive Unternehmen } \\
\text { ohne Kollaboration in der analysierten Periode. Die Analyse nimmt auch Bezug auf Unterschiede } \\
\text { quer durch verschiedene Zweige, verschiedene Arten von Unternehmungen und } \\
\text { Forschungsinstituten. Effekte sind deutlicher in großen Unternehmen als in kleinen. Effekte fallen } \\
\text { ebenfalls deutlicher aus bei exportierenden im Vergleich zu nicht-exportierenden Unternehmen. } \\
\text { Letztlich steigen Effekte mit dem Level an Fertigkeiten innerhalb der Unternehmen. }\end{array}$ \\
\hline Fokus & Bildung und Akademiker (Masterstudenten/innen und Doktoranten) im Business-Sektor \\
\hline $\begin{array}{l}\text { Studie Nr. 7- } \\
9\end{array}$ & $\begin{array}{l}\text { Drei unabhängige Studien des dänischen Industrie-PhD Programmes (DASTI 2007, DASTI } \\
\text { 01/2011 und der Effekt der Industrie-Doktorat Programme auf Einkommen und Beschäftigung } \\
\text { 2013) zeigen positive, statistisch signifikante Effekte. Dabei wurden 200-300 teilnehmende } \\
\text { Unternehmen sowie } 400 \text { teilnehmende Forscher abhängig von den Key-Impact Indikatoren } \\
\text { analysiert. Das Programm gewährt Zuschüsse an Unternehmen, die einen PhD-Studenten zur } \\
\text { Durchführung eines Doktorat-Projekts anstellen. Key Performance Indikatoren bestehen aus } \\
\text { Arbeitsproduktivität, individueller Beschäftigung, Gesamt-Beschäftigung in Unternehmen, } \\
\text { Patentaktivität, individuellem Gehalt und Gesamt-Faktorproduktivität. Die Analyse von 01/2011 } \\
\text { zeigt positiven und statistisch signifikanten Impact für kleine und mittlere Unternehmen in } \\
\text { Hinsicht auf Arbeitsproduktivität, Patentaktivität und Beschäftigung im Vergleich zu statistisch } \\
\text { ähnlichen Unternehmen ohne Industrie-Doktorat Projekte. Die Patentaktivität hat sich dabei fast } \\
\text { verdoppelt und die Beschäftigung ist um } 2 \text { Anstellungen pro Doktorat-Projekt pro Jahr gewachsen. } \\
\text { Beide Analysen zeigen einen positiven Impact für individuelle Beschäftigung und Gehälter in } \\
\text { Unternehmen. Keine zeigt einen Impact auf die Gesamt-Faktorproduktivität oder auf größere } \\
\text { Unternehmen. 2012/2013 wurden auch andere Studien des Industrie-Doktorat Programmes über } \\
\text { Einkommens- und Beschäftigungseffekten durchgeführt. }\end{array}$ \\
\hline
\end{tabular}


Studie Nr. 10-11 und Studie Nr. 18 (1)

Studie Nr. 12

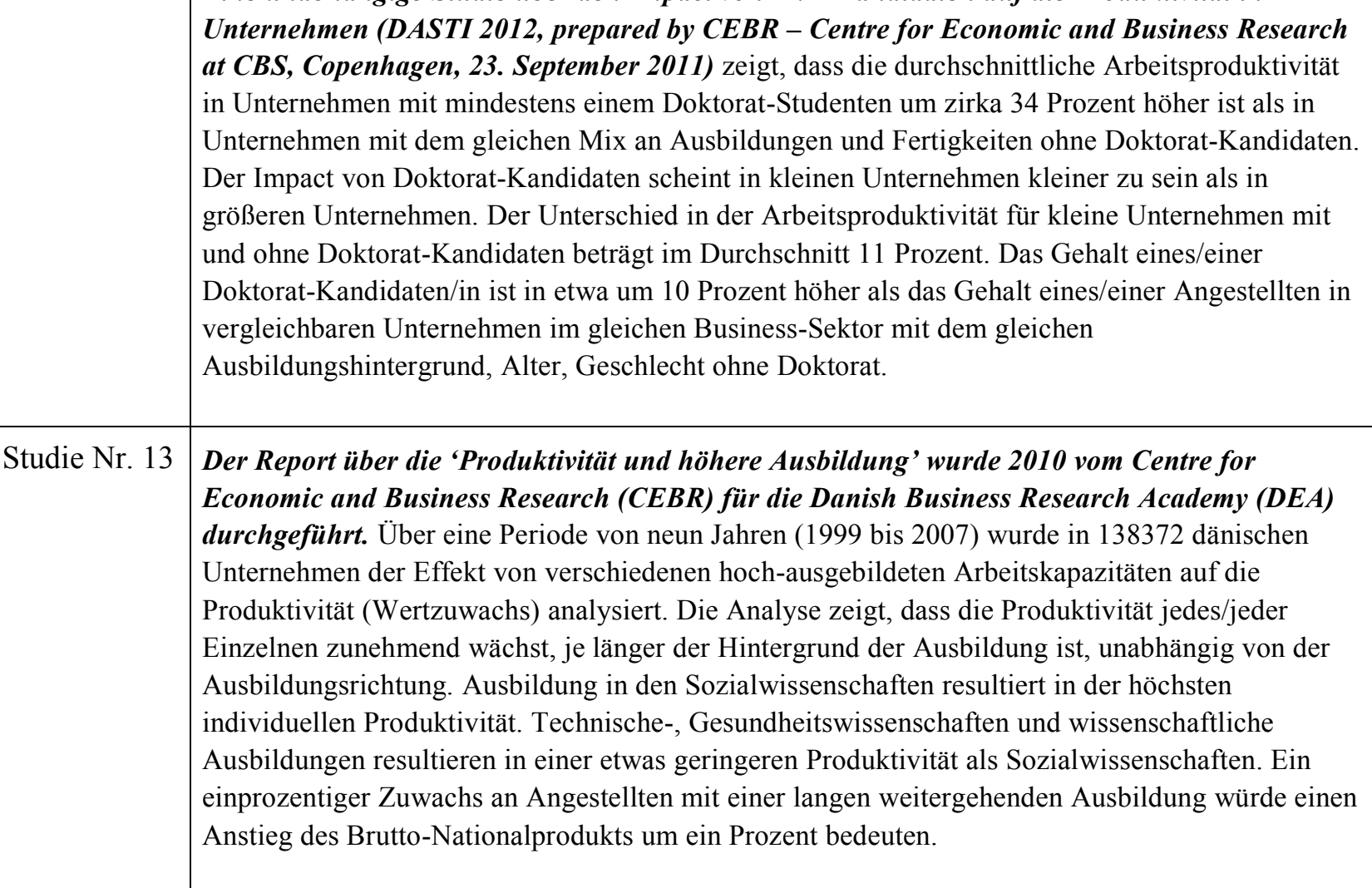

Eine unabhängige Impact Messung des dänischen Wissenspilot (Innovation Assistant) Programmes (DASTI 04/2010) zeigt positive aber statistisch nicht relevante Effekte für Unternehmen. Der Brutto-Gewinn stieg im Durchschnitt um 156000 EUR in den drei Jahren nachdem das Wissenspilot-Projekt gestartet wurde. Das Programm gewährt KMUs Zuschüsse bis zu 20000 EUR für das Anstellen von Akademikern. Die analysierten KPIs sind Brutto-Gewinn, Gesamt-Beschäftigung und Überlebensraten von Unternehmen. Auf Grund Fehlens von ausreichenden Daten und Beobachtungen wurde eine neue, unabhängige Messung durchgeführt.

\section{Eine Messung des dänischen Wissenspilot-Programms (Innovation Assistant Programme,} Videnpilotordningen) (DASTI 12/2013) zeigt positive Kurzzeit-Effekte auf die Beschäftigung für die Wissenspiloten, allerdings keine statistisch signifikanten Effekte für Unternehmen. Das Programm gewährleistet eine Förderung von bis zu 20000 EUR für KMUs für die Anstellung von Universitätsabsolventen. Die analysierten KPI sind Brutto-Gewinn, Wertzuwachs, Anlagenrendite, Arbeitsproduktivität, Gesamt-Beschäftigung und Überlebensraten von Unternehmen. Diese Ergebnisse werden von einer neuen Studie bestätigt (siehe Studie Nr. 18), welche zeigt, dass Unternehmen, die am Wissenspilotprogramm teilnehmen, hinsichtlich Faktorproduktivität in den zwei Jahren nach der Teilnahme im Vergleich zu ähnlichen nicht-teilnehmenden Unternehmen im Durchschnitt in etwa um 3 Prozentpunkte schneller wachsen.

Der Impact auf das Wachstum der Produktivität des dänischen Innovations- und Research Support Systems (DASTI 02/2014, April 2014): Eine unabhängige Meta-Messung (siehe Studie Nr. 18) entdeckte, dass Unternehmen, die am Wissenspilot-Programm teilgenommen haben, in den folgenden zwei Jahre hinsichtlich Gesamt-Faktorproduktivität im Durchschnitt um 2,9 Prozentpunkte schneller wachsen als ähnliche nicht-teilnehmende Unternehmen.

Eine

Eine unabhängige Studie über den Impact von PhD-Kandidaten auf die Produktivität in 


\begin{tabular}{|c|c|}
\hline Studie Nr. 14 & $\begin{array}{l}\text { Eine Analyse des makro-ökonomischen Gewinns der Anwerbung internationaler Studenten } \\
\text { (2013). Das dänische Ministerium für Forschung und weiterführende Bildung hat eine Analyse des } \\
\text { sozio-ökonomischen Gewinns der Anwerbung internaitonaler Studenten, die ein volles } \\
\text { Mastersprogramm in Dänemark absolvieren, in Auftrag gegeben. Die Analyse wurde von der } \\
\text { Danish Rational Economic Agents Model (DREAM) Gruppe durchgeführt. Die Analyse zeigt, dass } \\
\text { das Anwerben von internationalen Studenten mit einem Bachelor -Abschluss für ein volles } \\
\text { Mastersprogramm in Dänemark sozio-ökonomisch gewinnbringend ist. Dies gilt sowohl für jene } \\
\text { Studenten, die die Ausbildungskosten des Mastersprogrammes selbst finanzieren, als auch für } \\
\text { Studenten aus anderen EU/EEA Ländern, deren Mastersprogramm vom dänischen Staat finanziert } \\
\text { wird. Das Anwerben von } 1000 \text { zusätzlichen internationalen Studenten pro Jahr resultiert in der } \\
\text { bleibenden Verbesserung der öffentlichen Finanzen um zirka } 0.4 \text { bis } 0.8 \text { Milliarden DKK. Dabei } \\
\text { handelt es sich primär um Steuereinnahmen von Absolventen, die in Dänemark bleiben. Das } \\
\text { DREAM-Modell und alle Berechnungen basieren auf der Erfahrung von Konsumentenverhalten, } \\
\text { Unternehmen, etc. Eine wichtige Hypothese in diesen bestimmten Berechnungen ist, dass die } \\
\text { Anzahl an in Dänemark bleibenden Absolventen wie in den vergangenen Jahren gleich bleibt. }\end{array}$ \\
\hline Fokus & Kommerzieller Gewinn von öffentlicher und privater Forschung und Erfindungen \\
\hline Studie Nr. 15 & $\begin{array}{l}\text { Eine unabhängige Impact Messung des Inkubator-Programmes (DASTI 01/2010) zeigt keine } \\
\text { statistisch signifikanten Effekte für mehr als } 300 \text { Unternehmen und } 300 \text { Unternehmer. Das } \\
\text { Programm gewährt öffentliches Risikokapital für die Etablierung neuer wissensintensiver } \\
\text { Unternehmen. Die KPIs sind individuelle Gehälter, Gewinn, Wertzuwachst, Gesamt-Beschäftigung } \\
\text { und Überlebensrate der Unternehmen. Wegen Mangels an genügend Daten und Beobachtungen } \\
\text { wird } 2014 \text { eine neue unabhängige Impact Messung durchgeführt. Der Fokus dieser Studie liegt auf } \\
\text { den Effekten auf Unternehmensebene. }\end{array}$ \\
\hline Fokus & Meta-Messungen \\
\hline Stuc &  \\
\hline
\end{tabular}




\begin{tabular}{|c|c|}
\hline Studie Nr. 17 & $\begin{array}{l}\text { DASTI 01/2014), “Economic impacts of Business Investments in R\&D in the Nordic Countries - } \\
\text { A microeconomic analysis” schätzt Arbeitsproduktivitäts-Elastizitäten von FuE Investitionen. Die } \\
\text { erwägten FuE Arbeitsproduktivitäts-Elastizitäten sind positiv und signifikant für Unternehmen in } \\
\text { Dänemark, Finnland, Norwegen und Schweden. Des Weiteren ist die Größe der Elastizität } \\
\text { innerhalb der normalen Grenzen, wie in der Literatur beschrieben. Beim Vergleich von FuE } \\
\text { Elastizitäten in den nordischen Ländern fällt auf, dass Dänemark und Schweden mit } 0,091 \\
\text { beziehungsweise } 0,107 \text { die niedrigsten Elastizitäten aufweisen. Zwischen den beiden Ländern } \\
\text { wurde kein signifikanter Unterschied berechnet. Im Gegensatz dazu verzeichnen Norwegen und } \\
\text { Finnland die höchste FuE Arbeitsproduktivitäts-Elastizitäten mit } 0,129 \text { beziehungsweise 0,167. Im } \\
\text { Vergleich der nordischen Länder hinsichtlich Rentabiliät des letzten investierten Euros in } \\
\text { Business Fue sind Unterschiede erkennbar. Schwedische Unternehmen haben die niedrigste } \\
\text { Rentabilität auf den letzten investierten Euro in R\&D mit einer mittleren Rate von } 16,4 \text { Prozent. } \\
\text { Für Dänemark fällt diese Rate mit } 34,2 \text { Prozent im nordischen Vergleich am höchsten aus, gefolgt } \\
\text { von Finnland und Norwegen. Ein Versuch mit unterschiedlicher Rentabilität zeigt, dass die } \\
\text { geschätzten Rentabilitätsraten von Business FuE in den nordischen Ländern signifikant } \\
\text { unterschiedlich sind. Dieses Resultat zeigt, dass dänische im Vergleich mit schwedischen, } \\
\text { norwegischen und finnischen Unternehmen eine signifikant höhere marginale Rentabilität in } \\
\text { Business FuE erzielt. Der Report zeigt ebenfalls, dass sich die Resultate quer durch Business } \\
\text { Sektoren und Größe der Unternehmen signifikant unterscheiden. }\end{array}$ \\
\hline Studie Nr. 18 & $\begin{array}{l}\text { Der Impact auf das Wachstum der Produktivität des dänischen Innovations- und Research } \\
\text { Support Systems (DASTI 02/2014, April 2014) Die dänische Agentur für Wissenschaft, } \\
\text { Technologie und Innovation (DASTI) hat eine Analyse der gemeinsamen Effekte dänischer } \\
\text { Innovation und Forschung in Auftrag gegeben. Diese ist die erste ihrer Art, welche die } \\
\text { gemeinsame Abschätzung des ökonomischen Effekts dänischer Innovations- und } \\
\text { Forschungsprogramme auf das Wachstum der Gesamtfaktor-Produktivität von Unternehmen zum } \\
\text { Ziel hat. Die Analyse vergleicht das Wachstum der Produktivität von Unternehmen, die von einem } \\
\text { Programm Unterstützung erhalten haben mit ähnlichen Unternehmen, die keine Unterstützung } \\
\text { bekommen haben. Aus der Analyse wird ersichtlich, dass die an einem Programm teilnehmenden } \\
\text { Unternehmen in den auf die Teilnahme folgenden } 2 \text { Jahre im Durchschnitt um 2,5 Prozentpunkte } \\
\text { schneller gewachsen sind als nicht-teilnehmende Unternehmen. Des Weiteren zeigt die Analyse, } \\
\text { dass Programme (u.a. Innovation Network, Innovation Voucher), die darauf abzielen ein } \\
\text { Wissenswachstum via Kollaboration, Beratung und Unterstützung in Gang zu setzen, einen } \\
\text { Produktivitätszuwachs um } 2 \text { bis 3,75 Prozentpunkte bewirken. Die Schätzung basiert auf einer } \\
\text { Stichprobe von rund } 1100 \text { Beobachtungen in der Zeit } 2000 \text { bis 2011. Die Daten für die Stichprobe } \\
\text { wurden aus der Innovation Denmark Datenbank (siehe Seite 9) und angepassten Arbeiter- } \\
\text { Unternehmen Registrierungsdaten von Statistics Denmark zusammen genommen. }\end{array}$ \\
\hline
\end{tabular}

